\title{
Performance Evaluation of Gated Pipes Technique for Improving Surface Irrigation Efficiency in Maize Hybrids
}

\author{
Osama A. M. Ali ${ }^{*}$, Ahmed S. H. Mohammed ${ }^{2}$ \\ ${ }^{1}$ Crop Science Department, Faculty of Agriculture, Minufiya University, Shebin El-Kom, Egypt \\ ${ }^{2}$ Irrigation Department, Agricultural Engineering Research Institute, ARC, Giza, Egypt \\ Email: ${ }^{*}$ os ali2000@yahoo.com
}

Received 16 April 2015; accepted 25 May 2015; published 29 May 2015

Copyright (C) 2015 by authors and Scientific Research Publishing Inc.

This work is licensed under the Creative Commons Attribution International License (CC BY). http://creativecommons.org/licenses/by/4.0/

(c) $\underset{\mathrm{EY}}{\mathrm{F}}$ Open Access

\begin{abstract}
Waterlogging and low application efficiency are the main problems inherent with surface irrigation in the Nile Delta. Develop surface irrigation using gated pipes (GP) is a new method to be used to distribute water into furrow irrigated fields as strategy based on water saving. Laboratory calibration was conducted out to evaluate the hydraulic characteristics of pipe gates. Field experiments were conducted at the Experimental Farm of the Agriculture Faculty, Minufiya University during 2013 and 2014 seasons to evaluate the performance of utilize gated pipes technique for irrigating five maize varieties (S.C 10, S.C 130, S.C 131, S.C 2031 and T.W.C 321). The results revealed that the highest amount of water applied was with traditional surface irrigation $\left(6423.81 \mathrm{~m}^{3} \cdot \mathrm{ha}^{-1}\right)$. Use of gated pipes system GP1 as compared to traditional irrigation reduced water application by $923.81 \mathrm{~m}^{3} \cdot \mathrm{ha}^{-1}$ with grain and stover yields increases of $5.7 \%$ and $3.4 \%$, respectively. Traditional irrigation system achieved lowest irrigation performance parameters compared to gated pipes systems. Maize physiological attributes, yield, water use efficiency (WUE) and nitrogen accumulation were significantly decreased by either deficit or surplus irrigation than of GP1 rate. S.C 2031 variety significantly surpassed other varieties in abovementioned traits. Significant interaction effects were detected in both seasons. Maize varieties respond differently to irrigation systems. The highest values of grain yield (11062.6 and $\left.10911.8 \mathrm{~kg} \cdot \mathrm{ha}^{-1}\right)$ and stover yield (13639.0 and $13902.2 \mathrm{~kg} \cdot \mathrm{ha}^{-1}$ ) were obtained by S.C 2031 irrigated with GP1 system in both seasons. From the above mentioned results, it is concluded that the gated pipes technique is better than traditional irrigation for improving WUE and maize productivity under Nile Delta conditions.
\end{abstract}

\section{Keywords}

Maize, Hybrids, Gated Pipes, Irrigation Efficiency

\footnotetext{
${ }^{*}$ Corresponding author.
}

How to cite this paper: Ali, O.A.M. and Mohammed, A.S.H. (2015) Performance Evaluation of Gated Pipes Technique for Improving Surface Irrigation Efficiency in Maize Hybrids. Agricultural Sciences, 6, 550-570. 


\section{Introduction}

Maize (Zea mays L.) is one of the most important cereal crops grown principally during the summer season in Egypt. Great attention has been paid to increase maize total production. This could be achieved by using of high yielding varieties and avoid water flooding and deficit stress. [1] [2] reported that maize genotypes are significantly differed for grain and stover yields and associated traits. Many investigators found high variation among maize varieties in their yield in favor of single cross varieties as mentioned by [3] [4]. Meanwhile [5] found that three way cross 310 (T.W.C) significantly surpassed other single crosses (S.C 10 and S.C 122) in clay soil. The responses of plants to flooding or deficit water depend on the genotype, growth stage, level and duration of stress, and physical parameters of the soil. [6] [7] stated that plants are developed different morphological, physiological and biochemical mechanisms which inhibit the harmful effects of stresses.

Water scarcity is a growing global problem challenging sustainable development and placing a constraint on producing enough food to meet increasing food requirements. Egypt is mainly an agricultural country depending on the Nile water and consumes about $80 \%$ - $85 \%$ for agriculture annually [8]. The cultivated area of old land is about 2.7 million hectare irrigated by traditional surface irrigation (flooding method) according to data issued by Ministry of Agriculture, Egypt. Surface irrigation is the most widely used method as a conventional practice at the Egyptian farmers. Despite this progressive water shortage farmers continue to use flooding irrigation. Poor management, uniformity and distribution of water have been cited as the most frequent problems of flooding irrigation, resulting in waterlogging, salinization and less water use efficiency [9]. Water application efficiency gives a general sense of how well an irrigation system performs its primary task of getting water to the plant roots. Water infiltration into soil is a key to crop production and salinity control [10]. Researchers show that over $45 \%$ of water applied is lost to deep soil drainage and surface runoff [11].

The efficient use of water through modern irrigation systems is becoming increasingly important in arid and semi-arid regions with limited water resources [12]. Saving water and improving water use efficiency need to be developed. Water saved will be use for increase the cultivated area and overall crop production. Developing surface irrigation using gated pipes is a new method used to distribute water to furrow as strategy based on water saving. The gated pipe has many advantages: 1) requires small area of land to install the system; 2) reduces the seepage and evaporation losses and better water distribution; 3) low cost and maintenance requirements and 4) can improve human public health by avoiding contact with infected water. [13] reported that the differences between $0.80 \mathrm{ET}_{\mathrm{c}}$ and $1.00 \mathrm{ET}_{\mathrm{c}}$ treatments were not significant, while the lowest maize growth and yield were obtained from $0.60 \mathrm{ET}_{\mathrm{C}}$ treatment. The same authors added that the $0.80 \mathrm{ET}_{\mathrm{C}}$ irrigation treatment had the same or even greater WUE than 1.00 and $0.60 \mathrm{ET}_{\mathrm{c}}$. Nitrogen absorption by cereal crops plays a main role in plant growth. As a result of this, $\mathrm{N}$ is considered a strong tool for high crop yield. The excessive water application significantly reduced $\mathrm{N}, \mathrm{P}$ and $\mathrm{K}$ absorption of maize plants.

The main objectives of this study were to find out some practical effective ways regarding saving water particularly under the present status of water shortage facing Egypt. So, this study was planned to 1) improve the distribution uniformity of water discharge along the gated pipe and control of the water direction and reduce erosion in front of the gate, by designing a new outside gate locally to take the place of the side gate; and 2) evaluate the performance of some maize hybrids in light of use gated pipes technique for improving surface irrigation under the old lands of the Nile Delta.

\section{Materials and Methods}

\subsection{Experimental Site Specification}

\subsubsection{Laboratory Experiment}

Laboratory experiment was conducted at National Laboratory for Testing Irrigation Equipment, Agricultural Engineering Research Institute, Egypt to evaluate the hydraulic characteristics of a modified gate. The gate was designed from a gate valve $(3.45 \mathrm{~cm}$ D.) installed on the pipe orifice using rubber and external flexible hose mounted in front of the valve to control the direction of water. The laboratory testing equipments contained an electric centrifugal pump, flow meter, pressure gauges, manometer and control valve. The gate discharge along line was experimentally measured by direct method using bucket with capacity of 30 liter and stopwatch. Gated pipes line used for laboratory test was $12 \mathrm{~m}$ length with $150 \mathrm{~mm}$ inner diameter and $156 \mathrm{~mm}$ outer diameter. The velocity of water flow in the pipe was calculated according to the continuity equation, where discharge was 
measured by a flow meter. The distance between gates was fixed along the pipe line $(0.70 \mathrm{~m})$. Average discharge of water flowing in the gates was calculated by the following steps:

Calculation of the Reynold's number $\left(R_{e}\right)$ according to Equation (1) as given by [14].

$$
R_{e}=\frac{V \cdot D}{v}
$$

where:

$V=$ Average velocity in the pipe $\left(\mathrm{m} \cdot \mathrm{sec}^{-1}\right)$

$D=$ Inside pipe diameter $(\mathrm{m})$

$v=$ Kinematics viscosity $\left(\mathrm{m}^{2} \cdot \mathrm{sec}^{-1}\right)$

The used value of kinematic viscosity $(v)$ was taken $1 \times 10^{-6}$ considering that the water was at $20^{\circ} \mathrm{C}$.

Calculation of head losses due to friction from Equation (2)

$$
h_{f}=f \frac{L V^{2}}{D 2 g}
$$

where:

$$
\begin{aligned}
& h_{f}=\text { Friction head loss }(\mathrm{m}) \\
& f=\text { Darcy-Weisbach resistance coefficient } \\
& L=\text { Length of pipe }(\mathrm{m}) \\
& V=\text { Average velocity of water flowing in the pipe line }\left(\mathrm{m} \cdot \mathrm{sec}^{-1}\right) \\
& g=\text { Gravitational acceleration }\left(9.81 \mathrm{~m} \cdot \mathrm{sec}^{-2}\right) \\
& D=\text { Inner diameter of pipe }(\mathrm{m})
\end{aligned}
$$

The value of Darcy coefficient ( $f$ ) was calculated by using the Equation (3) consider that $R_{e}$ is up to $3.2 \times 10^{6}$.

$$
f=0.0032+\frac{0.221}{R_{e}^{0.237}}
$$

Calculation of head $\left(h_{s i}\right)$ which occurred due to the decrease in flow velocity inside the pipe was determined from Equation (4) as follows:

$$
h_{s i}=\frac{v_{\max }^{2}-v_{i}^{2}}{2 g}
$$

where:

$h_{s i}=$ Super imposed head (m)

$v_{\max }=$ Maximum average velocity of water flowing in the pipe line $\left(\mathrm{m} \cdot \mathrm{sec}^{-1}\right)$

$v_{i}=$ Average velocity of water flowing in the pipe line $\left(\mathrm{m} \cdot \mathrm{sec}^{-1}\right)$

Determination of the total head inside the pipe $\left(h_{m}\right)$ at any discharging outlet this was determined from Equation (5).

$$
h_{m}=h_{p}+h_{s i}-h_{f}
$$

where:

$h_{p}=$ Water head produced by the pump (m)

Considering the value of $h_{s i}$ is zero, the total head at the first gate (orifice) can be determined from Equation (6).

$$
h_{m 1}=h_{p}-h_{f}
$$

Determination of the average velocity $(v)$, the Equation (7) was used to calculate the average velocity at any gate (i)

$$
v_{i}=\sqrt{2 g h_{i}}
$$

Discharge of water at any gate was determined from Equation (8).

$$
q_{i}=c_{d} \times \frac{\pi}{4} d^{2} \times v
$$


where:

$q_{i}=$ Average discharge of water flowing in the gate $\left(\mathrm{m}^{3} \cdot \mathrm{sec}^{-1}\right)$

$c_{d}=$ Average coefficient of discharge $\left(c_{d}=0.65\right)$

$d=$ Average diameter of the gate $(\mathrm{m})$

$v=$ Average velocity in the gate $\left(\mathrm{m} \cdot \mathrm{sec}^{-1}\right)$

\subsubsection{Field Experiments}

Two field experiments were conducted at the Experimental Farm of the Faculty of Agriculture, Minufiya University in Shebin El-Kom, Egypt (latitude 30 31'39"N, longitude 31 $04^{\prime} 03^{\prime \prime E}$ ) during the two summer growing seasons of 2013 and 2014. Properties of the experimental soil are given in Table 1. Soil samples were randomly collected before sowing from depths of 0 - 20, 20 - 40 and $40-60 \mathrm{~cm}$ using an auger for estimating some mechanical and chemical properties as described by [15] [16]. Soil bulk density was determined using of cylinders according to [17]. Soil field capacity and wilting point were determined in the laboratory as described by [18].

\subsection{Experimental Design and Treatments}

The feasibility of producing maize under gated pipes technique was investigated comparing with traditional surface irrigation. The field experiment included twenty treatments which were all possible combinations between four irrigation treatments (traditional surface "flooding" and gated pipes with three discharge rates, i.e. 3.6 $\mathrm{m}^{3} \cdot \mathrm{h}^{-1}$ "GP1", $4.8 \mathrm{~m}^{3} \cdot \mathrm{h}^{-1}$ "GP2" and $6 \mathrm{~m}^{3} \cdot \mathrm{h}^{-1}$ "GP3") and five maize hybrids (S.C 10, S.C 130, S.C 131, S.C 2031 and T.W.C 321). The outside diameter of gated pipe is $6 "$ and $6 \mathrm{~m}$ length. Pipe is made of UPVC with gates spacing $0.70 \mathrm{~m}$. The flow rate out of each gate system is controlled by the percent of opening the main valve according to the discharge rates. Maize hybrids used for study were white color varieties produced by Agricultural Research Center, Egypt except S.C 2031 which produced by Hi-Tech Co.

A layout of the experimental plots is shown in Figure 1. Each of surface irrigation system (traditional or gated pipes) was divided into five sectors to evaluate the five hybrids which randomly assigned. Sub-plot area was $60 \mathrm{~m}$ length $\times 3.5 \mathrm{~m}$ width, occupying an area of $210 \mathrm{~m}^{2}$. Each plot was included 5 furrows, $0.7 \mathrm{~m}$ width for each (furrow area $=42 \mathrm{~m}^{2}$ ). A distance of $2.1 \mathrm{~m}$ was left between each irrigation treatments as a border. In the gated pipes technique, the pipes were located at the head of the irrigated field across the furrows and connected directly with main valve and water pump $\left(60 \mathrm{~m}^{3} \cdot \mathrm{h}^{-1}\right)$. Each furrow had one gate to water control and measure the irrigation efficiency. The irrigation treatments were distributed in the main plots, whereas maize hybrids were assigned at random in the sub-plots.

Table 1. (a) Mechanical and physical properties of the experimental field soil. (b) Chemical properties of the experimental field soil.

(a)

\begin{tabular}{|c|c|c|c|c|c|c|c|c|}
\hline \multirow{2}{*}{$\begin{array}{l}\text { Soil depth } \\
\text { (cm) }\end{array}$} & \multicolumn{3}{|c|}{ Particle size distribution (\%) } & \multirow{2}{*}{$\begin{array}{l}\text { Texture } \\
\text { class }\end{array}$} & \multirow{2}{*}{$\begin{array}{c}\text { Bulk density } \\
\mathrm{g} \cdot \mathrm{cm}^{-3}\end{array}$} & \multirow{2}{*}{$\begin{array}{c}\text { Field } \\
\text { capacity \% }\end{array}$} & \multirow{2}{*}{$\begin{array}{c}\text { Permanent } \\
\text { wilting point \% }\end{array}$} & \multirow{2}{*}{$\begin{array}{c}\text { Available soil } \\
\text { water } \%\end{array}$} \\
\hline & Sand & Silt & Clay & & & & & \\
\hline $0-20$ & 20.27 & 41.17 & 38.56 & Clay loam & 1.29 & 42.45 & 21.90 & 20.55 \\
\hline $20-40$ & 20.80 & 40.51 & 38.69 & Clay loam & 1.31 & 40.95 & 20.45 & 20.50 \\
\hline $40-60$ & 17.32 & 36.75 & 45.93 & Clay & 1.33 & 38.89 & 19.14 & 19.75 \\
\hline
\end{tabular}

(b)

\begin{tabular}{cccccccccccc}
\hline \multirow{2}{*}{$\begin{array}{c}\text { Soil depth } \\
(\mathbf{c m})\end{array}$} & \multicolumn{3}{c}{ Soluble cations meq·l } \\
\cline { 2 - 11 } & $\mathbf{N a}^{+}$ & $\mathbf{K}^{+}$ & $\mathbf{C a}^{2+}$ & $\mathbf{M g}^{2+}$ & $\mathbf{C l}^{-}$ & $\mathbf{H C O}_{3}^{2-}$ & $\mathbf{S O}_{4}^{2-}$ & $\mathbf{N}$ & $\mathbf{P}$ & $\mathbf{K}$ \\
\hline $0-20$ & 6.60 & 0.84 & 2.40 & 1.46 & 7.65 & 0.60 & 3.05 & 34.8 & 9.3 & 302.5 \\
$20-40$ & 4.85 & 0.70 & 1.53 & 1.42 & 5.75 & 0.45 & 2.30 & 32.4 & 8.4 & 287.1 \\
$40-60$ & 4.55 & 0.65 & 1.45 & 1.35 & 5.63 & 0.40 & 1.97 & 30.2 & 8.1 & 274.6 \\
\hline
\end{tabular}




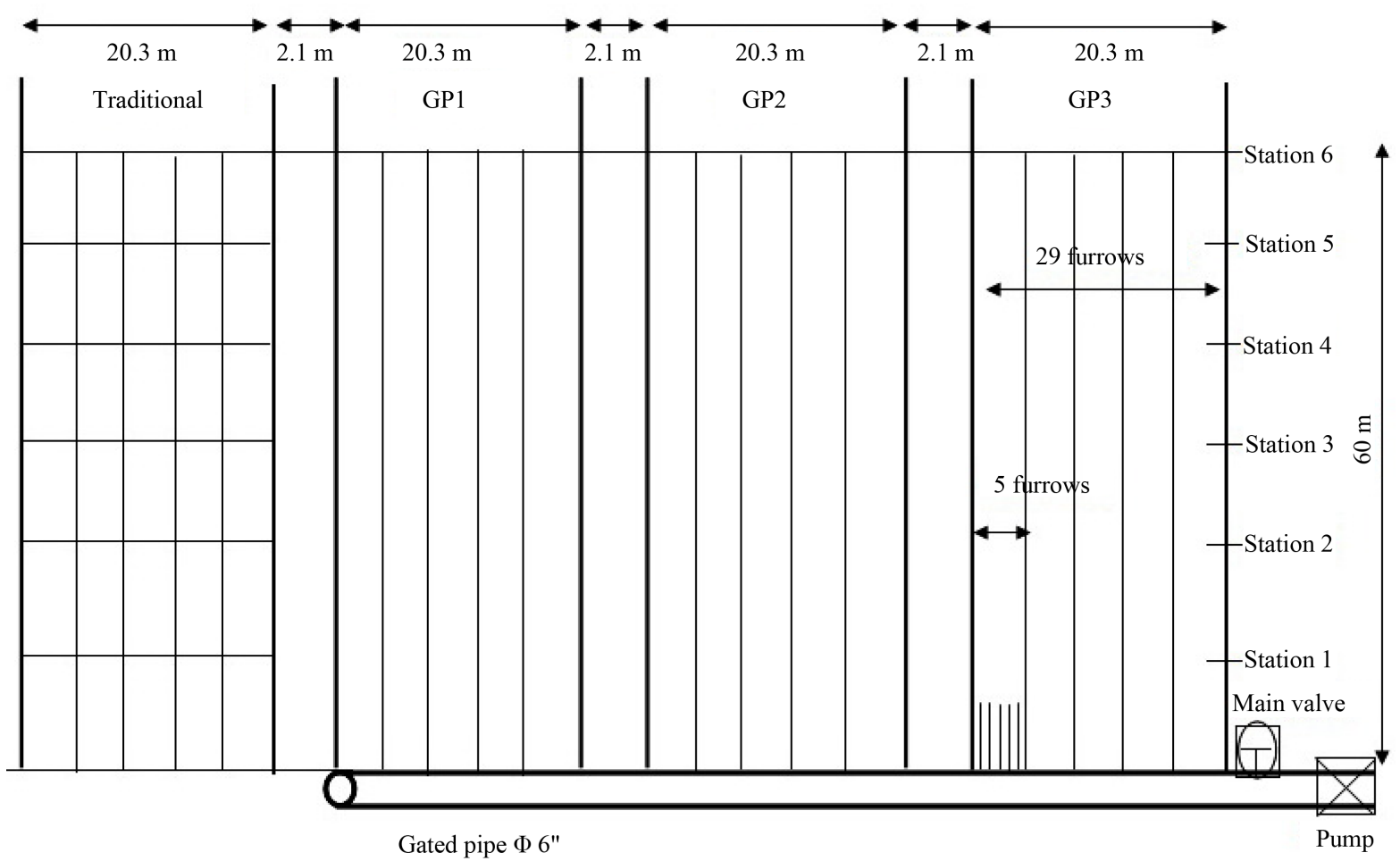

Figure 1. Layout of experimental replicate.

\subsection{Irrigation Performance Parameters}

Using field stalks and surveying tape, the furrows were divided into number of six stations having equal distances between them $(10 \mathrm{~m})$. Irrigation water advanced into the furrow (arrival times) were recorded at the end of each station. When water uncovers the field surface completely as a wave moving at the same direction of flow, recession times were observed and recorded at each station. This mark is the initiation of the water drying or recession front.

The water infiltration opportunity time along furrow length is the difference between the last time when water disappeared and the first time when water started at the same point along furrow. It can be determined according to [19] as formulated in Equation (9).

$$
t_{o}=t_{r}-t_{l}
$$

where:

$t_{o}=$ infiltration opportunity time (min)

$t_{l}=$ advance time (min)

$t_{r}=$ recession time (min)

Cutoff time of water flow (min $\cdot$ furrow $^{-1}$ ) is cumulative time since the initiation of irrigation until the inflow is terminated. It was recorded when the water has been totally arrived in the end of each furrow. Irrigation water applied $\left(\mathrm{m}^{3} \cdot \mathrm{ha}^{-1}\right)$ was calculated according to the cutoff time and discharge rate depends on the maize growth stage and environmental conditions.

Water infiltration in soil depth $(Z)$ was measured in the upper $30 \mathrm{~cm}$ of soil surface using double ring infiltrometer in beginning of experiment at site location. The two rings were driven into the soil to $15 \mathrm{~cm}$ depth. The two rings were set to measure infiltration rate in the next $15 \mathrm{~cm}$ soil layer. Filling water rate into inner cylinder was also recorded. The disappeared irrigation depth was recorded with interval time. Water infiltration was determined according to Equation (10).

$$
Z=9.32 t_{o}^{0.47}
$$

System coefficient of variation (CV\%) was estimated from Equation (11). 


$$
C V=\frac{S}{\bar{Z}}(100)
$$

where:

$S=$ Standard deviation

$\bar{Z}=$ Depth average of water distribution along furrow stations

The schedule parameter $(\alpha)$ specifies the deviation of any schedule irrigation depth $(d)$ to average of water distribution depth $(\bar{Z})$ in terms of CV and can be calculated according to [10] as formulated in Equation (12).

$$
\alpha=\frac{1}{C V}\left(\frac{d}{\bar{Z}}-1\right)
$$

where:

$d=$ Water depth expressing the plant water requirement calculated from ET .

The uniformity coefficient (UC) can be expressed in power distribution for water infiltrated depth which determined from Equation (13) as stated by [20].

$$
U C=1-0.8 C V
$$

The distribution uniformity (DU) is a measure of how uniformly water is applied to the area being watered, expressed as a percentage. It determined from Equation (14).

$$
D U=1-1.3 C V
$$

Application and storage efficiencies were used to evaluate the design of the irrigation system synchronizing with the irrigation scheduling.

Storage efficiency $\left(E_{s}\right)$ defined as the ratio of amount of water stored to the water needed into root zone. It was calculated as from Equation (15).

$$
E_{s}=100\left(1-P_{D}\right)
$$

The deficit percentage $\left(P_{D}\right)$ is defined as the ratio of water deficit to the required water into the root zone, can be formulated using linear distribution for water applied by the irrigation system (Figure 2) according to [21] in Equation (16).

$$
P_{D}=\frac{(1.725+\alpha)^{2} C V}{6.9(1+\alpha C V)}
$$

When the linear distribution is used to express the water profile of irrigation system, $\alpha$ will range from -1.725 to 1.725 under optimum irrigation, $\alpha \geq 1.725$ in deficit irrigation, and $\alpha \geq-1.725$ in excess irrigation according to [21]. In deficit irrigation condition, when $\alpha \geq 1.725$ and $d \geq Z_{\max }$, no deep seepage has occurred. The deficit percentage can be determined using of Equation (17).

\section{Fraction of area, $\mathrm{p}$}

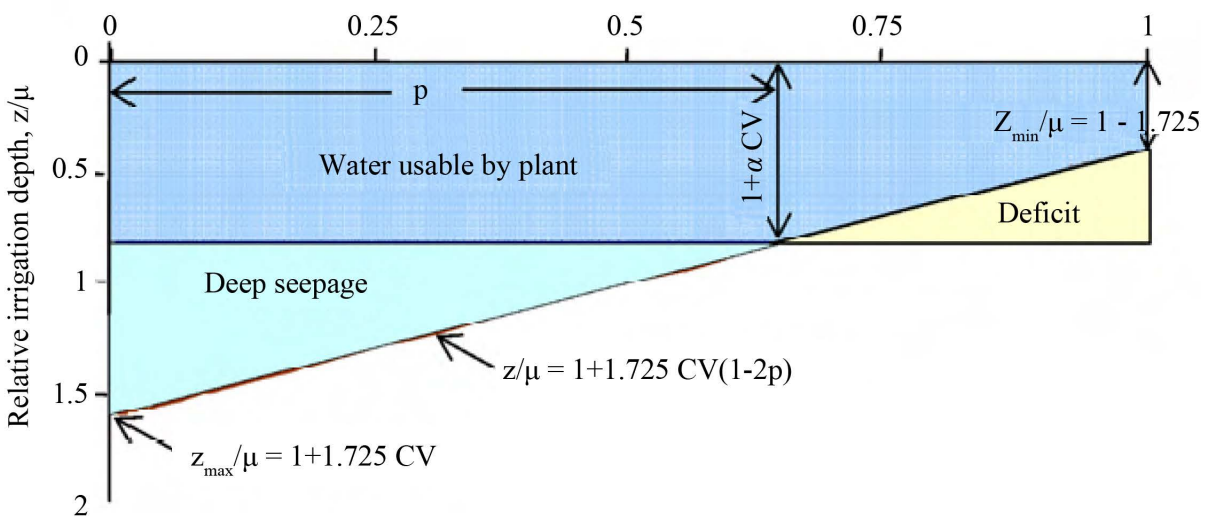

Figure 2. Linear cumulative frequency curve with relative required depth $(1+\alpha \mathrm{CV})$ according to [21]. 


$$
P_{D}=\frac{\alpha C V}{1+\alpha C V}
$$

Application efficiency $\left(E_{a}\right)$ is defined as the ratio of water stored in the root zone to the total water applied. $E_{a}$ was calculated from Equation (18).

$$
E_{a}=100\left(1-P_{s}\right)
$$

where:

$P_{s}=$ deep seepage percent.

The deep seepage percent can be described using a linear distribution as derivative from the basic analyses by [21]. In under irrigation when $\alpha$ ranged from -1.725 to 1.725 , deep seepage percentage $P_{s}$ could be determined from Equation (19).

$$
P_{s}=\frac{(1.725-\alpha)^{2} C V}{6.9}
$$

When $\alpha \leq-1.725$, deep seepage percentage could be calculated from Equation (20).

$$
P_{s}=-\alpha C V
$$

Water saving is referring to the consumption differences among surface irrigation systems.

\subsection{Agronomic Measurements}

During the growth phase, total chlorophyll (Chl) was measured at 50 days after sowing (DAS) with a hand-held chlorophyll meter (SPAD-502, Konica Minolta Company, Tokyo, Japan). At the period of 60 - 75 DAS crop growth rate (CGR) g plant ${ }^{-1}$ day $^{-1}$ was determined as the following formula:

$$
C G R=\frac{W_{2}-W_{1}}{T_{2}-T_{1}}
$$

where: $W_{1}$ and $W_{2}=$ plant dry weight (g.) at $T_{1}$ and $T_{2}$ (date of sampling), respectively. After 75 DAS, ear leaf area (ELA) was determined as formula, ELA $=$ ear blade length $\times$ maximum blade width $\times 0.75\left(\mathrm{~cm}^{2}\right)$.

At maturity, grain and stover yields (based on $15.5 \%$ moisture content) were determined by hand harvesting the area of two inner furrows and converted to $\mathrm{kg} \cdot \mathrm{ha}^{-1}$. Yield samples of eighteen plants were collected along the furrow from each plot to determine yield components.

Irrigation water use efficiency (WUE) in $\mathrm{kg} \cdot \mathrm{m}^{-3}$ was calculated according to formula, WUE $=$ Grain yield $\mathrm{kg} \cdot \mathrm{ha}^{-1}$ /amount of irrigation water applied $\mathrm{m}^{3} \cdot \mathrm{ha}^{-1}$.

Plant chemical composition was determined by plant partitioned into stover (stalk and leaves) and grains. Samples were dried in air-oven at $70^{\circ} \mathrm{C}$ to constant weight before grinding with a mill to pass through a $0.5 \mathrm{~mm}$ sieve. The samples were chemically analyzed to determine their contents of nitrogen. The total nitrogen percentage was determined by the Kjeldahl method as described by [22]. Nitrogen accumulation $\left(\mathrm{kg}^{\circ} \mathrm{ha}^{-1}\right)=$ sample $\mathrm{N}$ concentration $\times$ dry matter yield.

\subsection{Agronomic Practices}

The experimental field was ploughed twice, harrowed and leveled with slope $0.1 \%$ after wheat harvesting. Maize planting was done on $8^{\text {th }}$ and $10^{\text {th }}$ May 2013 and 2014, respectively using two grains per hill at a spacing of $25 \mathrm{~cm}$ and thinned to one plant at 21 DAS, to give a population of 57,140 plants ha ${ }^{-1}$. The experiment was irrigated seven times, where the first irrigation was applied at 21 DAS and the following irrigations were applied every 13 days until physiological maturity. All experimental plots were fertilized with NPK. Calcium superphosphate $\left(15.5 \% \mathrm{P}_{2} \mathrm{O}_{5}\right)$ was added during soil preparation at the rate of $74 \mathrm{~kg} \mathrm{P}_{2} \mathrm{O}_{5}$ ha $^{-1}$. Nitrogen fertilizer at a rate of $286 \mathrm{~kg} \mathrm{~N} \mathrm{ha}^{-1}$ in the form of urea $(46.5 \% \mathrm{~N})$ was added in two equal doses, the first dose was added after thinning (before the first irrigation), while the second dose was applied before the second irrigation. Potassium fertilizer was added in the form of potassium sulfate $\left(48 \% \mathrm{~K}_{2} \mathrm{O}\right)$ at the rate of $57 \mathrm{~kg} \mathrm{~K}_{2} \mathrm{O}$ ha ${ }^{-1}$ before the first irrigation. Weed control was done chemically before seedling emergence and mechanically after emergence in a timely manner. 


\subsection{Statistical Analysis}

Data were analyzed using an analysis of variance split plot design with three replicates described by [23]. Statistical analysis was done using CoStat Version 6.311 (CoHort software, USA). Treatments means were compared using Duncan's multiple test [24]. Means followed by the same letter are not significantly different from one another at $\mathrm{P}<0.05$. Capital letters in rows and columns indicate significant differences among irrigation systems and maize hybrids, respectively. Small letters indicate significant differences of the interaction between irrigation and hybrids treatments.

\section{Results and Discussion}

\subsection{Laboratory Calibration}

Laboratory calibration of modified gate along pipe line was illustrated in Figure 3. Results of hydraulic parameters (Table 2) showed that the average of determined discharges for GP1, GP2 and GP3 were 3.6, 4.8 and 6

Table 2. Hydraulic parameters of gates under different discharge rates.

\begin{tabular}{|c|c|c|c|c|c|c|c|c|c|}
\hline $\begin{array}{c}\text { Gate } \\
\text { number }\end{array}$ & $\begin{array}{c}Q \\
\left(\mathbf{m}^{3} \cdot \mathbf{h}^{-1}\right)\end{array}$ & $\begin{array}{c}\mathrm{V} \\
\left(\mathrm{m} \cdot \sec ^{-1}\right)\end{array}$ & $\mathbf{R}_{\mathbf{e}}$ & f & $\begin{array}{c}\mathbf{H}_{\mathbf{f}} \\
(\mathbf{m})\end{array}$ & $\begin{array}{l}\mathbf{H}_{\text {si }} \\
(\mathbf{m})\end{array}$ & $\begin{array}{l}\mathbf{H}_{\mathrm{m}} \\
(\mathbf{m})\end{array}$ & $\begin{array}{c}\mathrm{V}_{\mathrm{i}} \\
\left(\mathrm{m} \cdot \mathrm{sec}^{-1}\right)\end{array}$ & $\underset{\left(\mathbf{m}^{3} \cdot \mathbf{h}^{-1}\right)}{\mathbf{q}}$ \\
\hline \multicolumn{10}{|c|}{ GP1 system $\left(3.6 \mathrm{~m}^{3} \cdot \mathrm{h}^{-1}\right)$} \\
\hline 1 & 36.0 & 0.5657 & 84848.48 & 0.0182 & 0.0020 & 0.0000 & 0.1280 & 1.5849 & 3.4682 \\
\hline 2 & 32.4 & 0.5091 & 76363.64 & 0.0186 & 0.0028 & 0.0031 & 0.1303 & 1.5990 & 3.4992 \\
\hline 3 & 28.8 & 0.4525 & 67878.79 & 0.0190 & 0.0032 & 0.0059 & 0.1327 & 1.6136 & 3.5311 \\
\hline 4 & 25.2 & 0.3960 & 59393.94 & 0.0195 & 0.0032 & 0.0083 & 0.1351 & 1.6281 & 3.5628 \\
\hline 5 & 21.6 & 0.3394 & 50909.09 & 0.0201 & 0.0030 & 0.0104 & 0.1375 & 1.6422 & 3.5937 \\
\hline 6 & 18.0 & 0.2828 & 42424.24 & 0.0209 & 0.0026 & 0.0122 & 0.1397 & 1.6555 & 3.6228 \\
\hline 7 & 14.4 & 0.2263 & 33939.39 & 0.0218 & 0.0020 & 0.0137 & 0.1417 & 1.6676 & 3.6493 \\
\hline 8 & 10.8 & 0.1697 & 25454.55 & 0.0232 & 0.0013 & 0.0149 & 0.1435 & 1.6780 & 3.6722 \\
\hline 9 & 7.2 & 0.1131 & 16969.70 & 0.0252 & 0.0007 & 0.0157 & 0.1449 & 1.6864 & 3.6904 \\
\hline 10 & 3.6 & 0.0566 & 8484.85 & 0.0291 & 0.0002 & 0.0162 & 0.1459 & 1.6921 & 3.7029 \\
\hline \multicolumn{10}{|c|}{ GP2 system $\left(4.8 \mathrm{~m}^{3} \cdot \mathrm{h}^{-1}\right)$} \\
\hline 1 & 48.0 & 0.7542 & 113131.3 & 0.0172 & 0.0033 & 0.0000 & 0.2267 & 2.1089 & 4.6150 \\
\hline 2 & 43.2 & 0.6788 & 101818.2 & 0.0176 & 0.0047 & 0.0055 & 0.2308 & 2.1282 & 4.6572 \\
\hline 3 & 38.4 & 0.6034 & 90505.05 & 0.0180 & 0.0053 & 0.0104 & 0.2351 & 2.1478 & 4.7001 \\
\hline 4 & 33.6 & 0.5279 & 79191.92 & 0.0185 & 0.0054 & 0.0148 & 0.2394 & 2.1672 & 4.7426 \\
\hline 5 & 28.8 & 0.4525 & 67878.79 & 0.0190 & 0.0050 & 0.0186 & 0.2435 & 2.1859 & 4.7836 \\
\hline 6 & 24.0 & 0.3771 & 56565.66 & 0.0197 & 0.0043 & 0.0218 & 0.2475 & 2.2035 & 4.8221 \\
\hline 7 & 19.2 & 0.3017 & 45252.53 & 0.0206 & 0.0033 & 0.0244 & 0.2511 & 2.2194 & 4.8569 \\
\hline 8 & 14.4 & 0.2263 & 33939.39 & 0.0218 & 0.0022 & 0.0264 & 0.2542 & 2.2331 & 4.8868 \\
\hline 9 & 9.6 & 0.1508 & 22626.26 & 0.0237 & 0.0012 & 0.0279 & 0.2567 & 2.2440 & 4.9107 \\
\hline 10 & 4.8 & 0.0754 & 11313.13 & 0.0274 & 0.0004 & 0.0287 & 0.2583 & 2.2514 & 4.9268 \\
\hline \multicolumn{10}{|c|}{ GP3 system $\left(6 \mathrm{~m}^{3} \cdot \mathrm{h}^{-1}\right)$} \\
\hline 1 & 60.0 & 0.9428 & 141414.1 & 0.0165 & 0.0050 & 0.0000 & 0.3600 & 2.6577 & 5.8161 \\
\hline 2 & 54.0 & 0.8485 & 127272.7 & 0.0168 & 0.0041 & 0.0067 & 0.3647 & 2.6748 & 5.8534 \\
\hline 3 & 48.0 & 0.7542 & 113131.3 & 0.0172 & 0.0033 & 0.0126 & 0.3696 & 2.6930 & 5.8932 \\
\hline 4 & 42.0 & 0.6599 & 98989.90 & 0.0177 & 0.0026 & 0.0179 & 0.3748 & 2.7116 & 5.9340 \\
\hline 5 & 36.0 & 0.5657 & 84848.48 & 0.0182 & 0.0020 & 0.0224 & 0.3799 & 2.7301 & 5.9745 \\
\hline 6 & 30.0 & 0.4714 & 70707.07 & 0.0189 & 0.0014 & 0.0263 & 0.3849 & 2.7479 & 6.0134 \\
\hline 7 & 24.0 & 0.3771 & 56565.66 & 0.0197 & 0.0010 & 0.0294 & 0.3895 & 2.7643 & 6.0493 \\
\hline 8 & 18.0 & 0.2828 & 42424.24 & 0.0209 & 0.0006 & 0.0319 & 0.3935 & 2.7787 & 6.0807 \\
\hline 9 & 12.0 & 0.1886 & 28282.83 & 0.0227 & 0.0003 & 0.0336 & 0.3968 & 2.7903 & 6.1061 \\
\hline 10 & 6.0 & 0.0943 & 14141.41 & 0.0261 & 0.0001 & 0.0347 & 0.3991 & 2.7983 & 6.1237 \\
\hline
\end{tabular}




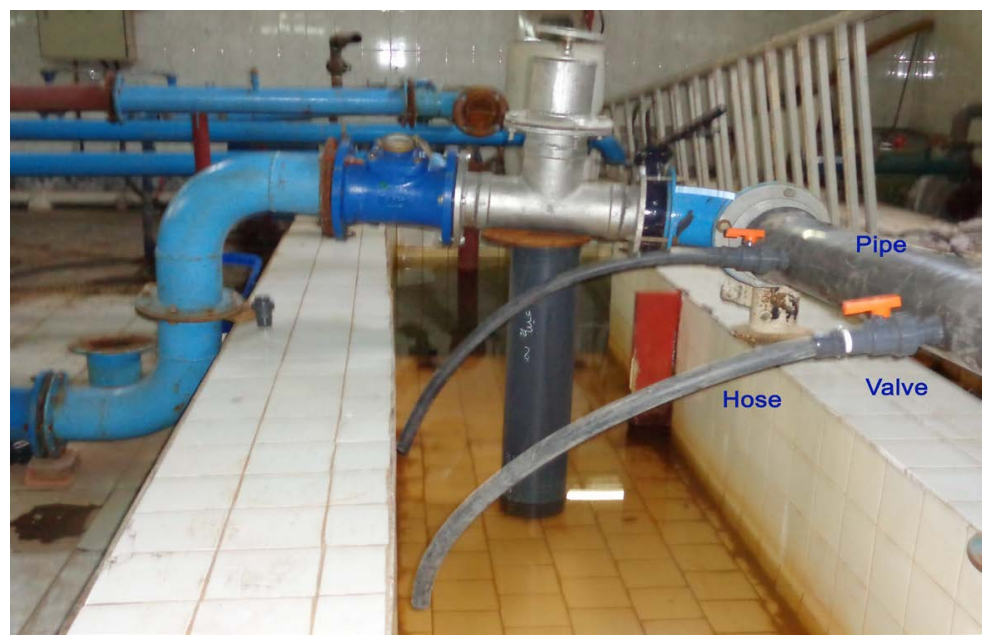

Figure 3. Laboratory calibration for gated pipe line.

$\mathrm{m}^{3} \cdot \mathrm{h}^{-1}$ respectively. The actual discharges (q) from the gates along line were nearly equal to theoretical discharges values. The coefficient of variation (CV\%) of discharges gates along the pipes line was $2.28 \%, 2.27 \%$ and $1.80 \%$ for GP1, GP2 and GP3 systems, respectively. The values of head pressure pump were 13, 23 and $36.5 \mathrm{~cm}$ for GP1, GP2 and GP3, respectively. This means that gates discharges were approximately constant. Accordingly, uniformity of water flow from the first gate to last gate along gated pipe lines according to different GP systems.

\subsection{Irrigation Performance Parameters}

\subsubsection{Water Saving}

Results in Table 3 show that the values of irrigation water applied varied from 4404.76 to $6423.81 \mathrm{~m}^{3} \cdot \mathrm{ha}^{-1}$ per season. Utilization of GP1, GP2 or GP3 as compared to traditional method reduced substantial amounts of water by $923.81,1566.67$ and $2019.05 \mathrm{~m}^{3} \cdot \mathrm{ha}^{-1}$, respectively. Water saving may be resulting from minimize advance time, which results in lowering water losses. Maize is a water-stress sensitive crop. If water saving is a major issue, then, some yield reduction must be accepted as shown by the trade-off in this study between water saving and yield loss under GP2 and GP3. Using a higher efficiency gated pipes irrigation system is recommended for irrigating maize especially under deficit irrigation in case of water scarcity from a water-saving viewpoint.

\subsubsection{Advance and Recession Phases}

Results of the advance and recession times and infiltration volume of the irrigation systems were illustrated in Figure 4 for fourth irrigation at 60 DAS. Under gated pipe irrigation technique, the highest advance and recession phase exhibited by GP3 $\left(6 \mathrm{~m}^{3} \cdot \mathrm{h}^{-1}\right)$ meanwhile the slow advance rate resulted from low water outflow (3.6 $\mathrm{m}^{3} \cdot \mathrm{h}^{-1}$ ). The advance phase should be completed as quickly as possible so that the intake opportunity time over the field will be uniform and then cutoff water inflow when enough water has been added to refill the root zone. This can be accomplished with a high water discharge into the field but without soil erosion. These results agree with those reported by [25] [26].

\subsubsection{Water Infiltration}

Infiltration rate as a function of opportunity time $t_{0}$ is illustrated in Figure 4 for fourth irrigation at 60 DAS. Infiltration is perhaps the most crucial factor affecting surface irrigation. The amount of water entering the soil and the duration of that irrigation varies greatly with irrigation systems. Because of this extreme variability, the infiltration rate is often varied along furrow. Water infiltration in traditional methods was greater than in gated pipes systems and it was basically due to the lower speed of water in the furrows. Furrows had satisfied their requirements of irrigation and some stations had over irrigation volumes in traditional method, resulting in more water deep seepage than other irrigation systems. Generally, GP1 (Figure 4(b)) achieved the best water infiltration with less water deficit. Meanwhile, GP3 (Figure 4(d)) produced more water deficit than other irrigation 
Table 3. Cutoff time and amount of water applied per each irrigation (average of the two seasons).

\begin{tabular}{|c|c|c|c|c|c|c|c|}
\hline \multirow{3}{*}{$\begin{array}{l}\text { Irrigation } \\
\text { number }\end{array}$} & \multirow{3}{*}{$\begin{array}{c}\begin{array}{c}\text { Traditional } \\
\text { irrigation }\end{array} \\
\text { Water applied } \\
\quad\left(\mathbf{m}^{3} \cdot \mathbf{h a}^{-1}\right)\end{array}$} & \multicolumn{6}{|c|}{ Gated pipes discharge rates } \\
\hline & & \multicolumn{2}{|c|}{ GP1 $\left(3.6 \mathrm{~m}^{3} \cdot \mathrm{h}^{-1}\right)$} & \multicolumn{2}{|c|}{ GP2 $\left(4.8 \mathrm{~m}^{3} \cdot \mathrm{h}^{-1}\right)$} & \multicolumn{2}{|c|}{ GP3 $\left(6 \mathrm{~m}^{3} \cdot \mathrm{h}^{-1}\right)$} \\
\hline & & $\begin{array}{l}\text { Cutoff } \\
\text { time } \\
\text { (min.) }\end{array}$ & $\begin{array}{c}\text { Water } \\
\text { applied } \\
\left(\mathbf{m}^{3} \cdot \mathbf{h a}^{-1}\right)\end{array}$ & $\begin{array}{l}\text { Cutoff } \\
\text { time } \\
\text { (min.) }\end{array}$ & $\begin{array}{c}\text { Water } \\
\text { applied } \\
\left(\mathrm{m}^{3} \cdot \mathrm{ha}^{-1}\right)\end{array}$ & $\begin{array}{l}\text { Cutoff } \\
\text { time } \\
\text { (min.) }\end{array}$ & $\begin{array}{c}\text { Water applied } \\
\left(\mathbf{m}^{3} \cdot \mathbf{h a}^{-1}\right)\end{array}$ \\
\hline 1 & 880.95 & 55 & 785.71 & 36 & 685.71 & 26 & 619.05 \\
\hline 2 & 861.90 & 52 & 742.86 & 35 & 666.67 & 25 & 595.24 \\
\hline 3 & 907.14 & 53 & 757.14 & 32 & 609.52 & 26 & 619.05 \\
\hline 4 & 1023.81 & 61 & 871.43 & 45 & 857.14 & 32 & 761.90 \\
\hline 5 & 1050.00 & 63 & 900.00 & 44 & 838.10 & 28 & 666.67 \\
\hline 6 & 857.14 & 53 & 757.14 & 32 & 609.52 & 24 & 571.43 \\
\hline 7 & 842.86 & 48 & 685.71 & 31 & 590.48 & 24 & 571.43 \\
\hline Sum & 6423.81 & 385 & 5500.00 & 255 & 4857.14 & 185 & 4404.76 \\
\hline
\end{tabular}

systems due to little quantity of water applied. These results are consistent with the findings of [21] [25].

\subsubsection{Application and Storage Efficiencies}

Data in Table 4 show that application efficiency was improved by delivering water inside the furrow in gated pipes systems to minimize advance time, which results in decreasing water losses and ensuring that the depths and discharge variations over the field are relatively uniform and, as a result, available soil water in the root zone is also uniform. Traditional and GP1 irrigation systems achieved the highest storage efficiency without significant difference. Storage efficiency achieved values equal or nearly $100 \%$ in complete excess irrigation condition because the root zone is fully irrigated $\left(P_{D}=0\right)$, while application efficiency $\left(E_{a}\right)$ have values less than $100 \%$ depending on uniformity CV [10]. Water application efficiency gives a general sense of how well an irrigation system performs its primary task of getting water to the plant roots. According to previous results, there is strong evidence that GP2 followed by GP1systems are more efficient than traditional irrigation method. In this respect, [26] indicated that the gated-pipe system has a high value of application efficiency $(79 \%-88 \%)$ compared with the open field head ditch (69\% - 71\%).

\subsubsection{Uniformity}

From the data in Table 4, it is evident that the values of distribution uniformity and uniformity coefficient were significantly influenced by irrigation systems. Gated pipes systems exhibited the highest values of DU and UC more than traditional method. Uniformity of water distribution in GP3 system was better than the other irrigation systems. The low value of uniformity was mainly due to the longer contact time which leads to temporal variations of the soil moisture distribution which is more evident along the field irrigated with traditional method. Non-uniformity of water application under irrigation system creates both deficit and excessive irrigation amounts into plant root zone [21]. To achieve high efficiency and uniformity of surface irrigation systems, all parts of an irrigated field should receive water for near equal period of time with a minimum of water lost to runoff or to deep percolation below the root zone.

\subsection{Physiological Attributes}

Physiological traits were significantly affected by irrigation systems and maize hybrids (Table 5 ). In both seasons, GP1 $\left(3.6 \mathrm{~m}^{3} \cdot \mathrm{h}^{-1}\right)$ exhibited the highest values of Chl, CGR and ELA. The increases in Chl, CGR and ELA resulting from GP1 amounted to $14.34 \%, 13.44 \%$ and $5.46 \%$, respectively more than the traditional irrigation. Flooding irrigation treatment was over-irrigated and resulted in considerable water losses by runoff and deep percolation below the root zone (Figure 4). Relative greenness of maize leaves was also affected due to flooding treatment as there was fading of leaf color as reflected by their corresponding SPAD values [27]. In this concern, [28] [29] mentioned that increasing or decreasing soil moisture may be resulted in unbalanced soil water-air re- 
O. A. M. Ali, A. S. H. Mohammed
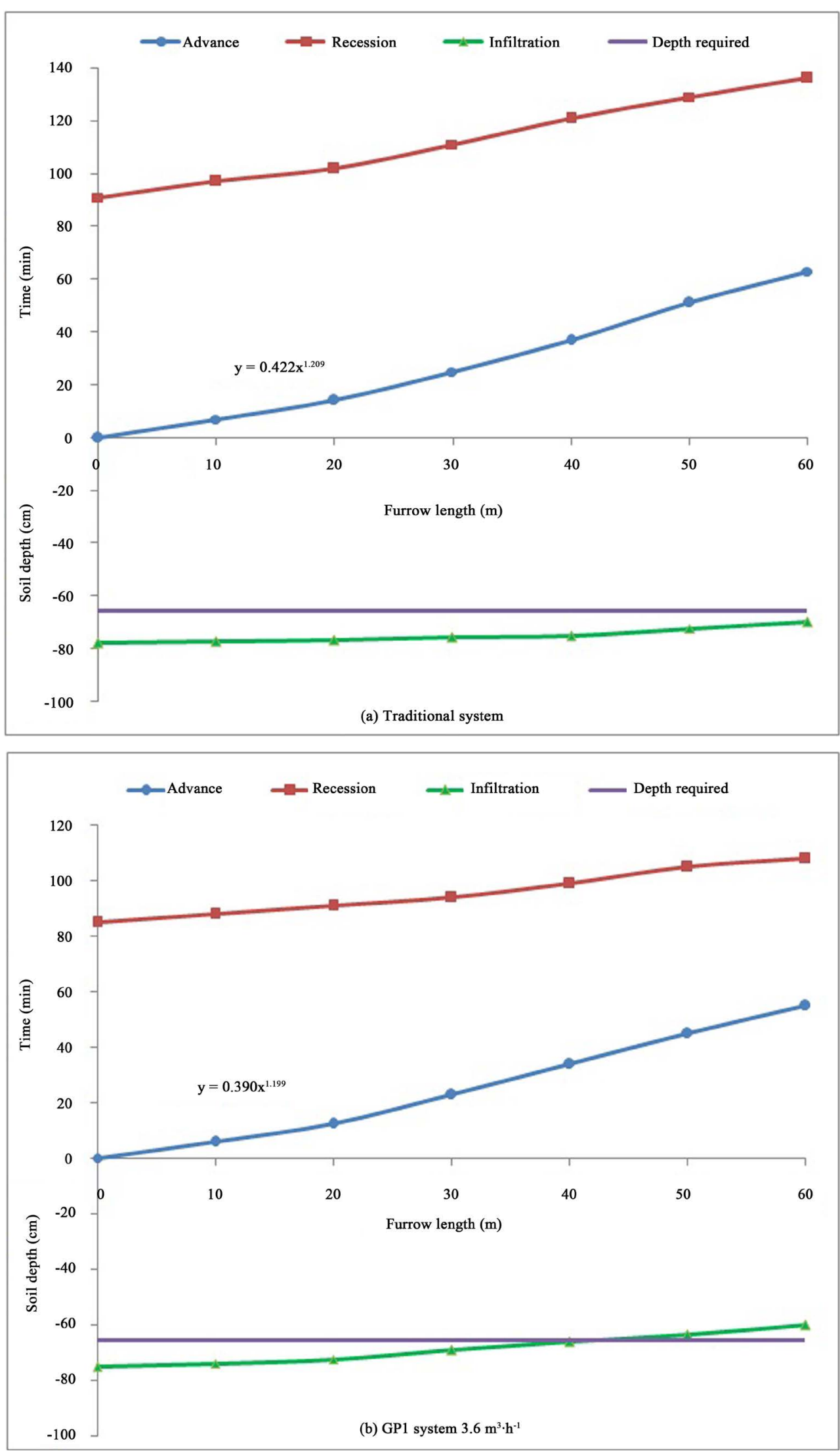

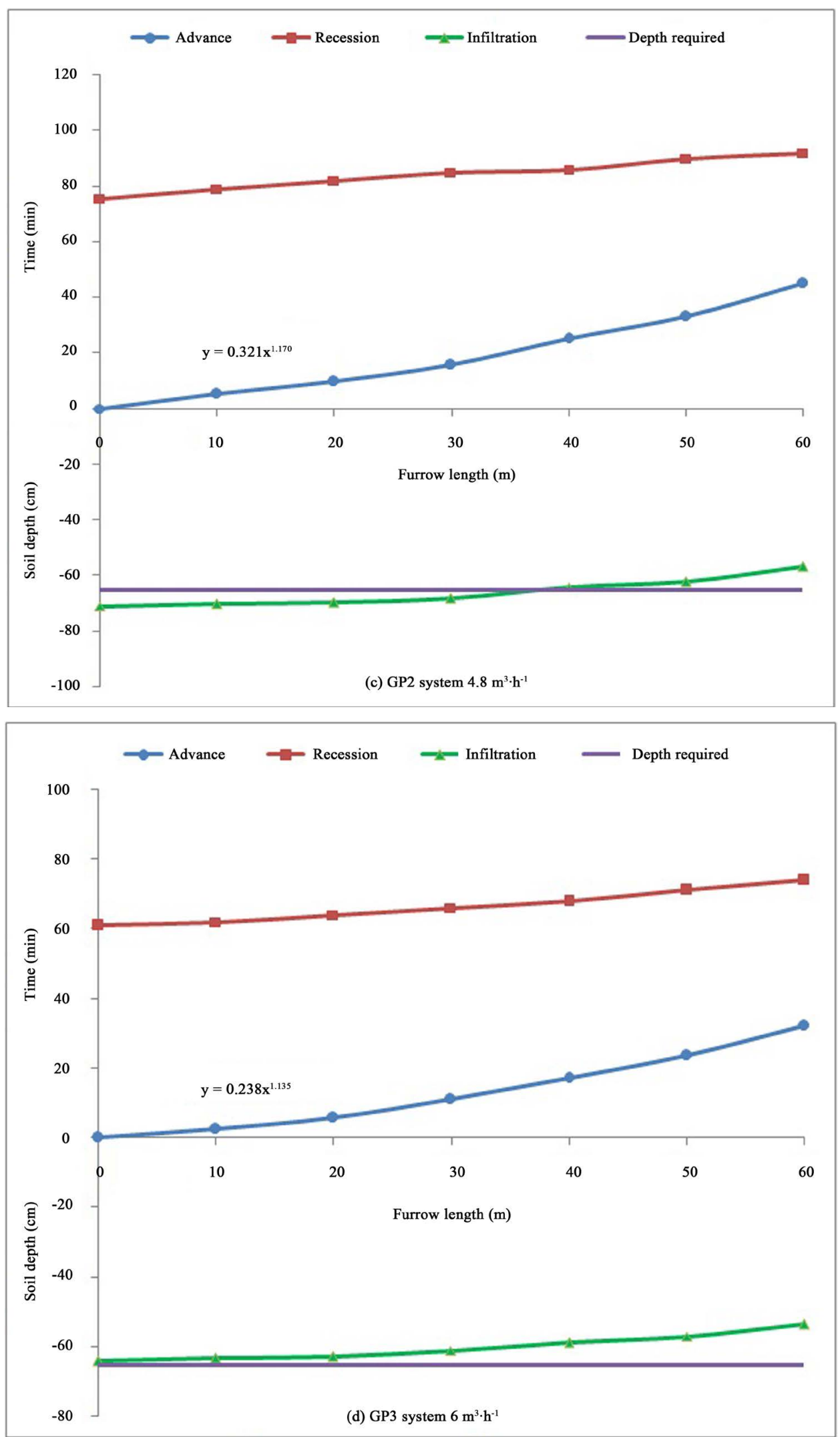

Figure 4. Advance, recession and infiltrated water distributions curves of irrigation systems. 
Table 4. Irrigation performance parameters per each irrigation (average of the two seasons).

\begin{tabular}{|c|c|c|c|c|c|c|c|}
\hline $\begin{array}{l}\text { Irrigation } \\
\text { number }\end{array}$ & Ź (cm) & CV\% & $\alpha$ & UC\% & DU\% & $\mathbf{E}_{\mathrm{a}} \%$ & $E_{s} \%$ \\
\hline \multicolumn{8}{|c|}{ Traditional system } \\
\hline 1 & 72.738 & 10.901 & -0.976 & 91.279 & 85.829 & 88.475 & 99.008 \\
\hline 2 & 72.374 & 10.871 & -2.996 & 91.304 & 85.868 & 67.428 & 100.00 \\
\hline 3 & 73.051 & 10.785 & -1.276 & 91.372 & 85.979 & 89.089 & 99.634 \\
\hline 4 & 73.984 & 10.395 & -1.090 & 91.684 & 86.487 & 91.679 & 99.315 \\
\hline 5 & 76.945 & 12.070 & -0.532 & 90.344 & 84.310 & 94.357 & 97.341 \\
\hline 6 & 72.374 & 10.871 & 0.715 & 91.304 & 85.868 & 99.068 & 91.296 \\
\hline 7 & 71.829 & 10.746 & 0.255 & 91.404 & 86.031 & 98.201 & 94.055 \\
\hline Mean & 73.328 a & 10.948 a & $-0.843 \mathrm{c}$ & $91.241 \mathrm{~d}$ & $85.767 d$ & 89.757 c & $97.236 \mathrm{a}$ \\
\hline \multicolumn{8}{|c|}{ GP1 system $\left(3.6 \mathrm{~m}^{3} \cdot \mathrm{h}^{-1}\right)$} \\
\hline 1 & 67.616 & 10.145 & -0.381 & 91.884 & 86.811 & 96.131 & 97.238 \\
\hline 2 & 66.681 & 9.602 & -2.793 & 92.318 & 87.518 & 73.184 & 100.00 \\
\hline 3 & 66.815 & 10.155 & -0.562 & 91.876 & 86.798 & 92.301 & 97.889 \\
\hline 4 & 69.446 & 11.035 & -0.502 & 91.172 & 85.655 & 92.070 & 97.467 \\
\hline 5 & 70.296 & 11.170 & 0.217 & 91.064 & 85.479 & 96.319 & 94.039 \\
\hline 6 & 66.815 & 10.155 & 1.649 & 91.876 & 86.798 & 99.991 & 85.652 \\
\hline 7 & 65.820 & 8.628 & 1.405 & 93.098 & 88.784 & 99.872 & 89.073 \\
\hline Mean & $67.641 \mathrm{~b}$ & $10.127 \mathrm{~b}$ & -0.138 bc & $91.898 \mathrm{c}$ & 86.835 c & $92.838 \mathrm{ab}$ & 94.480 a \\
\hline \multicolumn{8}{|c|}{ GP2 system $\left(4.8 \mathrm{~m}^{3} \cdot \mathrm{h}^{-1}\right)$} \\
\hline 1 & 63.563 & 6.448 & 0.351 & 94.842 & 91.618 & 98.235 & 96.063 \\
\hline 2 & 61.711 & 7.312 & -2.861 & 94.150 & 90.494 & 79.078 & 100.00 \\
\hline 3 & 60.340 & 6.439 & 0.685 & 94.849 & 91.629 & 98.990 & 95.777 \\
\hline 4 & 66.477 & 7.786 & -0.169 & 93.771 & 89.878 & 95.950 & 97.233 \\
\hline 5 & 66.524 & 7.517 & 1.095 & 93.986 & 90.228 & 99.568 & 92.395 \\
\hline 6 & 60.340 & 6.439 & 4.546 & 94.849 & 91.629 & 92.576 & 71.616 \\
\hline 7 & 59.870 & 6.035 & 3.855 & 95.172 & 92.155 & 96.031 & 77.905 \\
\hline Mean & $62.689 \mathrm{c}$ & $6.854 \mathrm{c}$ & $1.071 \mathrm{~b}$ & 94.517 b & $91.090 \mathrm{~b}$ & 94.347 a & 90.141 b \\
\hline \multicolumn{8}{|c|}{ GP3 system $\left(6 \mathrm{~m}^{3} \cdot \mathrm{h}^{-1}\right)$} \\
\hline 1 & 59.323 & 4.696 & 2.038 & 96.243 & 93.895 & 99.933 & 91.266 \\
\hline 2 & 59.294 & 4.288 & -4.127 & 96.570 & 94.425 & 82.301 & 100.00 \\
\hline 3 & 59.323 & 4.696 & 1.320 & 96.243 & 93.895 & 99.888 & 94.163 \\
\hline 4 & 60.340 & 6.439 & 1.354 & 94.849 & 91.629 & 99.872 & 91.981 \\
\hline 5 & 59.082 & 4.979 & 4.392 & 96.017 & 93.528 & 94.869 & 82.058 \\
\hline 6 & 59.349 & 3.570 & 8.804 & 97.144 & 95.360 & 74.077 & 76.089 \\
\hline 7 & 59.350 & 3.570 & 6.821 & 97.144 & 95.360 & 86.564 & 80.419 \\
\hline Mean & $59.437 \mathrm{~d}$ & $4.605 \mathrm{~d}$ & $2.943 \mathrm{a}$ & 96.316 a & $94.013 \mathrm{a}$ & 91.072 bc & 87.997 b \\
\hline
\end{tabular}


Table 5. Physiological traits as affected by surface irrigation systems and maize hybrids during 2013 and 2014 seasons.

\begin{tabular}{|c|c|c|c|c|c|c|c|c|c|c|c|c|}
\hline \multirow{2}{*}{$\begin{array}{c}\text { Hybrids } \\
\text { irrigation } \\
\text { systems }\end{array}$} & \multicolumn{6}{|c|}{2013} & \multicolumn{6}{|c|}{2014} \\
\hline & $\begin{array}{c}\text { SC } \\
10\end{array}$ & $\begin{array}{l}\text { SC } \\
130\end{array}$ & $\begin{array}{l}\text { SC } \\
131\end{array}$ & $\begin{array}{c}\text { SC } \\
2031\end{array}$ & $\begin{array}{c}\text { TWC } \\
321\end{array}$ & Mean & $\begin{array}{c}\text { SC } \\
10\end{array}$ & $\begin{array}{l}\text { SC } \\
130\end{array}$ & $\begin{array}{l}\text { SC } \\
131\end{array}$ & $\begin{array}{c}\text { SC } \\
2031\end{array}$ & $\begin{array}{c}\text { TWC } \\
321\end{array}$ & Mean \\
\hline & \multicolumn{12}{|c|}{ Total chlorophyll (SPAD value) } \\
\hline Traditional & $\begin{array}{c}38.41 \\
j\end{array}$ & $\begin{array}{c}39.64 \\
\text { ij }\end{array}$ & $\begin{array}{c}40.87 \\
\text { hi }\end{array}$ & $\begin{array}{c}43.41 \\
\text { fg }\end{array}$ & $\begin{array}{l}46.61 \\
\mathrm{~d}\end{array}$ & $\begin{array}{c}41.79 \\
\text { C }\end{array}$ & $\begin{array}{c}39.17 \\
\text { hij }\end{array}$ & $\begin{array}{c}41.04 \\
\text { fgh }\end{array}$ & $\begin{array}{c}38.05 \\
\text { ijk }\end{array}$ & $\begin{array}{c}41.67 \\
\text { fg }\end{array}$ & $\begin{array}{l}41.23 \\
\text { fgh }\end{array}$ & $\begin{array}{c}40.23 \\
\text { C }\end{array}$ \\
\hline GP1 & $\begin{array}{c}42.50 \\
\mathrm{~g}\end{array}$ & $\begin{array}{c}44.29 \\
\text { ef }\end{array}$ & $\begin{array}{c}45.31 \\
\text { de }\end{array}$ & $\begin{array}{c}54.48 \\
\mathrm{a}\end{array}$ & $\begin{array}{c}48.44 \\
\mathrm{C}\end{array}$ & $\begin{array}{c}47.01 \\
\text { A }\end{array}$ & $\begin{array}{l}43.37 \\
\text { ef }\end{array}$ & $\begin{array}{l}45.47 \\
\text { cde }\end{array}$ & $\begin{array}{c}47.38 \\
\text { bc }\end{array}$ & $\begin{array}{c}51.83 \\
\mathrm{a}\end{array}$ & $\begin{array}{c}45.79 \\
\text { cd }\end{array}$ & $\begin{array}{c}46.77 \\
\text { A }\end{array}$ \\
\hline GP2 & $\begin{array}{c}38.27 \\
j\end{array}$ & $\begin{array}{c}42.10 \\
\text { gh }\end{array}$ & $\begin{array}{c}40.24 \\
\mathrm{i}\end{array}$ & $\begin{array}{c}52.32 \\
\text { b }\end{array}$ & $\begin{array}{c}43.32 \\
\text { fg }\end{array}$ & $\begin{array}{c}43.25 \\
\text { B }\end{array}$ & $\begin{array}{c}40.22 \\
\text { ghi }\end{array}$ & $\begin{array}{c}40.81 \\
\text { gh }\end{array}$ & $\begin{array}{c}42.04 \\
\mathrm{fg}\end{array}$ & $\begin{array}{c}48.46 \\
b\end{array}$ & $\begin{array}{c}44.73 \\
\text { de }\end{array}$ & $\begin{array}{c}43.25 \\
\text { B }\end{array}$ \\
\hline GP3 & $\begin{array}{c}33.46 \\
\mathrm{k}\end{array}$ & $\begin{array}{c}40.15 \\
\mathrm{i}\end{array}$ & $\begin{array}{c}38.22 \\
j\end{array}$ & $\begin{array}{c}45.30 \\
\text { de }\end{array}$ & $\begin{array}{c}39.55 \\
\text { ij }\end{array}$ & $\begin{array}{c}39.34 \\
\text { D }\end{array}$ & $\begin{array}{c}36.42 \\
\mathrm{k}\end{array}$ & $\begin{array}{c}37.94 \\
j k\end{array}$ & $\begin{array}{l}41.19 \\
\text { fgh }\end{array}$ & $\begin{array}{c}42.00 \\
\text { fg }\end{array}$ & $\begin{array}{c}40.78 \\
\text { gh }\end{array}$ & $\begin{array}{c}39.67 \\
\text { C }\end{array}$ \\
\hline \multirow[t]{2}{*}{ Mean } & $\begin{array}{c}38.16 \\
\text { D }\end{array}$ & $\begin{array}{c}41.55 \\
\text { C }\end{array}$ & $\begin{array}{c}41.16 \\
C\end{array}$ & $\begin{array}{c}48.88 \\
\text { A }\end{array}$ & $\begin{array}{c}44.48 \\
\text { B }\end{array}$ & & $\begin{array}{c}39.80 \\
\text { D }\end{array}$ & $\begin{array}{c}41.32 \\
C\end{array}$ & $\begin{array}{c}42.17 \\
\text { BC }\end{array}$ & $\begin{array}{c}45.99 \\
\text { A }\end{array}$ & $\begin{array}{c}43.13 \\
\text { B }\end{array}$ & \\
\hline & \multicolumn{12}{|c|}{ Crop growth rate (g plant ${ }^{-1}$ day $^{-1}$ ) } \\
\hline Traditional & $\begin{array}{c}7.69 \\
\mathrm{ijk}\end{array}$ & $\begin{array}{c}8.51 \\
\text { fg }\end{array}$ & $\begin{array}{c}9.25 \\
\text { de }\end{array}$ & $\begin{array}{c}11.02 \\
\mathrm{~b}\end{array}$ & $\begin{array}{l}8.80 \\
\text { ef }\end{array}$ & $\begin{array}{c}9.05 \\
\text { B }\end{array}$ & $\begin{array}{c}8.12 \\
\mathrm{H}\end{array}$ & $\begin{array}{c}10.02 \\
\mathrm{e}\end{array}$ & $\begin{array}{c}9.36 \\
\mathrm{f}\end{array}$ & $\begin{array}{c}12.14 \\
\mathrm{~b}\end{array}$ & $\begin{array}{c}9.28 \\
\mathrm{f}\end{array}$ & $\begin{array}{c}9.78 \\
\text { B }\end{array}$ \\
\hline GP1 & $\begin{array}{c}8.24 \\
\mathrm{f}-\mathrm{i}\end{array}$ & $\begin{array}{c}10.21 \\
\mathrm{C}\end{array}$ & $\begin{array}{c}11.82 \\
\mathrm{a}\end{array}$ & $\begin{array}{c}12.27 \\
\mathrm{a}\end{array}$ & $\begin{array}{c}9.73 \\
\text { cd }\end{array}$ & $\begin{array}{c}10.46 \\
\text { A }\end{array}$ & $\begin{array}{c}8.84 \\
\mathrm{~g}\end{array}$ & $\begin{array}{c}11.20 \\
\text { C }\end{array}$ & $\begin{array}{c}10.62 \\
\mathrm{~d}\end{array}$ & $\begin{array}{c}12.74 \\
\mathrm{a}\end{array}$ & $\begin{array}{c}11.10 \\
\mathrm{C}\end{array}$ & $\begin{array}{c}10.90 \\
\text { A }\end{array}$ \\
\hline GP2 & $\begin{array}{c}7.34 \\
\mathrm{k}\end{array}$ & $\begin{array}{c}7.50 \\
\mathrm{jk}\end{array}$ & $\begin{array}{c}8.55 \\
\text { fg }\end{array}$ & $\begin{array}{c}9.24 \\
\text { de }\end{array}$ & $\begin{array}{l}8.28 \\
\text { fgh }\end{array}$ & $\begin{array}{c}8.18 \\
C\end{array}$ & $\begin{array}{c}6.66 \\
\mathrm{i}\end{array}$ & $\begin{array}{c}8.73 \\
\mathrm{~g}\end{array}$ & $\begin{array}{c}9.53 \\
\mathrm{f}\end{array}$ & $\begin{array}{c}10.13 \\
\mathrm{e}\end{array}$ & $\begin{array}{c}8.66 \\
\mathrm{~g}\end{array}$ & $\begin{array}{c}8.74 \\
\text { C }\end{array}$ \\
\hline GP3 & $\begin{array}{c}6.44 \\
1\end{array}$ & $\begin{array}{l}7.54 \\
\text { jk }\end{array}$ & $\begin{array}{l}7.96 \\
g-j\end{array}$ & $\begin{array}{c}8.66 \\
\mathrm{f}\end{array}$ & $\begin{array}{l}7.80 \\
\text { h-k }\end{array}$ & $\begin{array}{c}7.68 \\
\mathrm{D}\end{array}$ & $\begin{array}{c}6.04 \\
j\end{array}$ & $\begin{array}{c}8.08 \\
h\end{array}$ & $\begin{array}{c}8.57 \\
\mathrm{~g}\end{array}$ & $\begin{array}{c}9.49 \\
\mathrm{f}\end{array}$ & $\begin{array}{c}8.07 \\
\mathrm{~h}\end{array}$ & $\begin{array}{c}8.05 \\
\text { D }\end{array}$ \\
\hline \multirow[t]{2}{*}{ Mean } & $\begin{array}{c}7.43 \\
\text { D }\end{array}$ & $\begin{array}{c}8.44 \\
C\end{array}$ & $\begin{array}{c}9.40 \\
\text { B }\end{array}$ & $\begin{array}{c}10.30 \\
\text { A }\end{array}$ & $\begin{array}{c}8.65 \\
\text { C }\end{array}$ & & $\begin{array}{c}7.41 \\
\text { D }\end{array}$ & $\begin{array}{c}9.51 \\
\text { B }\end{array}$ & $\begin{array}{c}9.52 \\
\text { B }\end{array}$ & $\begin{array}{c}11.12 \\
\text { A }\end{array}$ & $\begin{array}{c}9.28 \\
\text { C }\end{array}$ & \\
\hline & \multicolumn{12}{|c|}{ Ear leaf area $\left(\mathrm{cm}^{2}\right)$} \\
\hline Traditional & $\begin{array}{c}571.5 \\
\mathrm{~g}\end{array}$ & $\begin{array}{c}587.3 \\
\text { f }\end{array}$ & $\begin{array}{c}631.5 \\
\text { c }\end{array}$ & $\begin{array}{c}681.3 \\
\text { b }\end{array}$ & $\begin{array}{c}588.5 \\
\mathrm{f}\end{array}$ & $\begin{array}{c}\text { 612.0 } \\
\text { B }\end{array}$ & $\begin{array}{c}594.6 \\
\mathrm{~g}\end{array}$ & $575.0 \mathrm{~h}$ & $\begin{array}{c}623.4 \\
\text { de }\end{array}$ & $\begin{array}{c}659.2 \\
\text { b }\end{array}$ & $\begin{array}{c}602.4 \\
\mathrm{fg}\end{array}$ & $\begin{array}{c}610.9 \\
\text { B }\end{array}$ \\
\hline GP1 & $\begin{array}{c}618.5 \\
\mathrm{~d}\end{array}$ & $\begin{array}{c}603.3 \\
\mathrm{e}\end{array}$ & $\begin{array}{c}686.9 \\
\text { b }\end{array}$ & $\begin{array}{c}710.9 \\
\mathrm{a}\end{array}$ & $\begin{array}{c}622.4 \\
\mathrm{~cd}\end{array}$ & $\begin{array}{c}648.4 \\
\text { A }\end{array}$ & $\begin{array}{c}630.3 \\
\mathrm{~d}\end{array}$ & $\begin{array}{c}618.4 \\
\mathrm{e}\end{array}$ & $\begin{array}{c}624.2 \\
\text { de }\end{array}$ & $\begin{array}{c}687.5 \\
\mathrm{a}\end{array}$ & $\begin{array}{c}646.2 \\
\text { c }\end{array}$ & $\begin{array}{c}641.3 \\
\text { A }\end{array}$ \\
\hline GP2 & $\begin{array}{c}531.2 \\
\mathrm{i}\end{array}$ & $\begin{array}{c}511.3 \\
\mathrm{j}\end{array}$ & $\begin{array}{c}597.4 \\
\text { ef }\end{array}$ & $\begin{array}{c}604.5 \\
\mathrm{e}\end{array}$ & $\begin{array}{c}563.4 \\
\mathrm{~g}\end{array}$ & $\begin{array}{c}561.6 \\
\text { C }\end{array}$ & $\begin{array}{c}552.4 \\
\mathrm{i}\end{array}$ & $\begin{array}{c}556.1 \\
\mathrm{i}\end{array}$ & $\begin{array}{c}603.9 \\
\mathrm{f}\end{array}$ & $\begin{array}{c}620.9 \\
\text { e }\end{array}$ & $\begin{array}{c}554.3 \\
\mathrm{i}\end{array}$ & $\begin{array}{c}577.5 \\
\text { C }\end{array}$ \\
\hline GP3 & $\begin{array}{c}440.3 \\
1\end{array}$ & $\begin{array}{c}476.2 \\
\mathrm{k}\end{array}$ & $\begin{array}{c}567.1 \\
\mathrm{~g}\end{array}$ & $\begin{array}{c}548.2 \\
\mathrm{~h}\end{array}$ & $\begin{array}{c}511.4 \\
\mathrm{j}\end{array}$ & $\begin{array}{c}508.6 \\
\text { D }\end{array}$ & $\begin{array}{c}461.5 \\
\mathrm{~m}\end{array}$ & $\begin{array}{c}496.9 \\
1\end{array}$ & $\begin{array}{c}513.4 \\
\mathrm{k}\end{array}$ & $\begin{array}{c}531.2 \\
\mathrm{j}\end{array}$ & $\begin{array}{c}536.3 \\
j\end{array}$ & $\begin{array}{c}507.9 \\
\text { D }\end{array}$ \\
\hline Mean & $\begin{array}{c}540.4 \\
\text { D }\end{array}$ & $\begin{array}{c}544.5 \\
\text { D }\end{array}$ & $\begin{array}{c}620.7 \\
\text { B }\end{array}$ & $\begin{array}{c}636.2 \\
\mathrm{~A}\end{array}$ & $\begin{array}{c}571.4 \\
\text { C }\end{array}$ & & $\begin{array}{c}559.7 \\
\text { D }\end{array}$ & $\begin{array}{c}561.6 \\
\text { D }\end{array}$ & $\begin{array}{c}591.2 \\
\text { B }\end{array}$ & $\begin{array}{c}624.7 \\
\text { A }\end{array}$ & $\begin{array}{c}584.8 \\
\text { C }\end{array}$ & \\
\hline
\end{tabular}

lations that lead to reducing the photosynthesis activity and unbalanced relations between plant hormones and biological processes in the whole plant organs. These adverse conditions in the treated soils are undoubtedly of great importance throughout the vegetative growth and dry matter accumulation. The findings obtained in this study were in good agreement to those reported by [30]-[33].

There were significant differences among maize hybrids in their physiological characters (Table 5). S.C 2031 variety significantly surpassed the other hybrids. S.C 2031 had Chl, CGR and ELA values that were 21.69\%, $44.34 \%$ and $14.62 \%$ greater than those of S.C 10 , respectively. Such results could be attributed to the differences in the genetic constitution of the tested varieties. Increase in Chl and consequently increase in rate of dry matter accumulation leads to an increase in ear leaf area because proportion of dry matter allocated to leaves are increased constantly. Genetic variability of maize genotypes was also reported by [2] [4] [27].

The interaction between irrigation systems and varieties had a significant effect on physiological traits in both seasons. S.C 2031 plants irrigated with GP1 $\left(3.6 \mathrm{~m}^{3} \cdot \mathrm{h}^{-1}\right)$ surpassed other combinations and gave the highest values of Chl (54.48 and 51.83), CGR (12.27 and 12.74) and ELA (710.88 and 687.45) in both seasons. Meanwhile, S.C 10 irrigated with GP3 $\left(6 \mathrm{~m}^{3} \cdot \mathrm{h}^{-1}\right)$ achieved the lowest values. Some of the differences among genotypes in growth and photosynthesis can be traced to different capacities for water acquisition and transport at a 
given water status [6]. This superiority might be due to well utilization of soil nutrients in meristematic tissues and metabolism activity which improved these traits. These results are consistent with the findings of [27] and [32].

\subsection{Yield and Its Components}

The data in Table 6 and Table 7 show that the differences among the surface irrigation treatments were significant for yield and its components. The irrigation systems significantly affected plant height. Using of GP1 system $\left(3.6 \mathrm{~m}^{3} \cdot \mathrm{h}^{-1}\right)$ achieved the longest stems, while the shortest one was recorded with GP3 system $\left(6 \mathrm{~m}^{3} \cdot \mathrm{h}^{-1}\right)$. With regard to maize ear traits, GP1 system surpassed other systems in number of rows per ear, number of grains per row and 100-grain weight followed by traditional irrigation in both seasons. From these results, it can be concluded that the superiority of GP1 in yield than traditional irrigation may be generally due to the tendency of this technique to produce more leaf chlorophyll, ear leaf area and CGR as shown in Table 5. The maize productivity was highly significantly affected by irrigation systems. Gated pipes system (GP1) produced the highest significant grain and stover yields, followed by traditional system. In contrast, GP3 system produced the lowest yields in both seasons. These results showed a positive relation between the yield and water quantity applied (Table 3). However, applying several inches of excess water (flooding) will lower the net return for the irrigated field potentially due to depressed grain yield resulting from more infiltration in traditional irrigation than GP (Figure 4) lead to leaching nutrients below the active root zone and inhibiting soil aeration. On the contrary, water deficit reduce carbon availability and dry matter partitioning to the ear during the critical period that determines grain number [34] and size of the sinks [1]. Corn yield is closely related to crop evapotranspiration $\left(\mathrm{ET}_{\mathrm{c}}\right)$ and usually yield would be lowered if ET is lowered [12]. Drought and flooding are abiotic stresses which caused crop yield decrease [21] [35]. In this concern, other investigators indicated that the furrow irrigation by gated pipes achieved the highest yields of maize [9] [36] and sugarcane [26].

Results in Table 6 and Table 7 indicated that maize varieties exhibited significant differences in their yield and its components. Results revealed that, S.C 2031 variety significantly surpassed other varieties in plant height followed by T.W.C 321 variety, while S.C 130 had the shortest plants. S.C 2031 variety recorded the highest values of number of rows per ear, number of grains per row and plant grain yield without significant different with S.C 130 and S.C 131 for number of rows in the first season. However, the highest grain index was recorded by T.W.C 321 followed by S.C 2031 variety without significant. On the contrary, the lowest values were obtained by S.C 10 (for number of rows per ear), S.C 130 (for 100-grain weight) and S.C 131 (for number of grains per row) in both seasons. These results can be attributable to changes in light interception and utilization due to increases in chlorophyll content and ear leaf area (Table 5). An increase in CGR during the vegetative growth is indicative of response of the photosynthetic apparatus to an increase in demand for assimilates to afford growth of the grain fraction. Varieties significantly affected dry matter production and partitioning into the different plant components (grain and stover). S.C 2031 exhibited the highest grain and stover yields ha ${ }^{-1}$ in both seasons. It is clear from the obtained results that, S.C 2031 variety sowing led to an increase amounted to $32.06 \%, 21.71 \%, 12.29 \%$ and $18.99 \%$ for grain yield ha $^{-1}$ and $39.44 \%, 21.75 \%, 11.09 \%$ and $20.71 \%$ for stover yield ha ${ }^{-1}$ than other S.C 10, S.C 130, S.C 131 and T.W.C 321, respectively. Genotypic superiority for grain and stover yields is particularly related to differences in any of yield components and dependent on the inherent genetic potential of the varieties themselves. In our study, varieties with higher values of physiological traits were higher yielding than those with lower values. These results are in harmony with those obtained by [4] [37] and [38] who indicated that single crosses of maize significantly surpassed the other crosses in growth and yield attributes.

The interaction effect between irrigation systems and varieties had a significant effect on the most yield and its components traits. No interaction effects were detected on number of rows per ear and 100-grain weight in both seasons. The highest values of yield attributes being obtained for S.C 2031 variety irrigated with GP1. By contrast, the shortest plants were recorded by S.C 130 and S.C 10 varieties irrigated with GP3. The lowest grains number was detected by irrigated S.C 10 and S.C 130 in the first season and S.C 10, S.C 130 and S.C 131 in the second one with GP3 system. Concerning maize yields, S.C 2031 variety irrigated with GP1 exhibited the first significant rank in plant grain yield and grain and stover yields ha ${ }^{-1}$. Therefore this combination is recommended as the treatment that maximizes grain and stover yields. This combination may be exhibited better water and light utilization due to maintenance of green leaf area and leaf photosynthesis rather than other treatments. There is evidence that the variations in the grain yield response were due to variations in physiological traits that co- 
Table 6. Yield components of maize as affected by surface irrigation systems and maize hybrids during 2013 and 2014 seasons.

\begin{tabular}{|c|c|c|c|c|c|c|c|c|c|c|c|c|}
\hline \multirow{2}{*}{$\begin{array}{c}\text { Hybrids } \\
\text { irrigation } \\
\text { systems }\end{array}$} & \multicolumn{6}{|c|}{2013} & \multicolumn{6}{|c|}{2014} \\
\hline & $\begin{array}{l}\text { SC } \\
10\end{array}$ & $\begin{array}{l}\text { SC } \\
130\end{array}$ & $\begin{array}{l}\text { SC } \\
131\end{array}$ & $\begin{array}{c}\text { SC } \\
2031\end{array}$ & $\begin{array}{c}\text { TWC } \\
321\end{array}$ & Mean & $\begin{array}{l}\text { SC } \\
10\end{array}$ & $\begin{array}{c}\text { SC } \\
130\end{array}$ & $\begin{array}{l}\text { SC } \\
131\end{array}$ & $\begin{array}{c}\text { SC } \\
2031\end{array}$ & $\begin{array}{c}\text { TWC } \\
321\end{array}$ & Mean \\
\hline & \multicolumn{12}{|c|}{ Plant height (cm) } \\
\hline Traditional & $\begin{array}{c}270.5 \\
\text { ef }\end{array}$ & $\begin{array}{c}250.6 \\
\text { hi }\end{array}$ & $\begin{array}{c}279.4 \\
\text { cde }\end{array}$ & $\begin{array}{c}289.8 \\
\text { abc }\end{array}$ & $\begin{array}{c}283.5 \\
\text { bcd }\end{array}$ & $\begin{array}{c}274.8 \\
\text { B }\end{array}$ & $\begin{array}{c}276.2 \\
\text { c }\end{array}$ & $\begin{array}{c}246.2 \\
\text { de }\end{array}$ & $\begin{array}{c}289.3 \\
\text { b }\end{array}$ & $\begin{array}{c}294.3 \\
\text { ab }\end{array}$ & $\begin{array}{c}290.9 \\
\text { b }\end{array}$ & $\begin{array}{c}279.4 \\
\text { B }\end{array}$ \\
\hline GP1 & $\begin{array}{c}278.4 \\
\text { de }\end{array}$ & $\begin{array}{c}256.2 \\
\text { gh }\end{array}$ & $\begin{array}{c}287.3 \\
\text { bcd }\end{array}$ & $\begin{array}{c}300.1 \\
\mathrm{a}\end{array}$ & $\begin{array}{c}292.6 \\
\text { ab }\end{array}$ & 282.9 A & $\begin{array}{c}275.7 \\
\text { C }\end{array}$ & $\begin{array}{c}251.5 \\
d\end{array}$ & $\begin{array}{c}301.5 \\
\text { ab }\end{array}$ & $\begin{array}{c}305.8 \\
\mathrm{a}\end{array}$ & $\begin{array}{c}292.0 \\
\text { b }\end{array}$ & $\begin{array}{c}285.3 \\
\text { A }\end{array}$ \\
\hline GP2 & $\begin{array}{c}234.2 \\
\text { ij }\end{array}$ & $\begin{array}{c}236.4 \\
j k\end{array}$ & $\begin{array}{c}263.8 \\
\text { fg }\end{array}$ & $\begin{array}{c}262.6 \\
\text { fg }\end{array}$ & $\begin{array}{c}255.0 \\
\text { ghi }\end{array}$ & $\begin{array}{c}250.4 \\
\text { C }\end{array}$ & $\begin{array}{c}239.0 \\
\text { ef }\end{array}$ & $\begin{array}{c}229.7 \\
\text { fgh }\end{array}$ & $\begin{array}{c}268.1 \\
\text { C }\end{array}$ & $\begin{array}{c}272.4 \\
\text { C }\end{array}$ & $\begin{array}{c}269.0 \\
\text { C }\end{array}$ & $\begin{array}{c}255.7 \\
\text { C }\end{array}$ \\
\hline GP3 & $\begin{array}{c}222.4 \\
1\end{array}$ & $\begin{array}{c}227.1 \\
\mathrm{kl}\end{array}$ & $\begin{array}{c}244.6 \\
\text { ij }\end{array}$ & $\begin{array}{c}253.1 \\
\text { fgh }\end{array}$ & $\begin{array}{c}248.9 \\
\text { hi }\end{array}$ & $239.2 \mathrm{D}$ & $\begin{array}{c}224.9 \\
\text { gh }\end{array}$ & $\begin{array}{c}218.6 \\
\text { h }\end{array}$ & $\begin{array}{c}237.1 \\
\text { efg }\end{array}$ & $\begin{array}{c}254.3 \\
d\end{array}$ & $\begin{array}{c}251.9 \\
\mathrm{~d}\end{array}$ & $\begin{array}{c}237.4 \\
\text { D }\end{array}$ \\
\hline \multirow[t]{2}{*}{ Mean } & $\begin{array}{c}251.4 \\
\text { C }\end{array}$ & $\begin{array}{c}242.6 \\
\text { D }\end{array}$ & $\begin{array}{c}268.8 \\
\text { B }\end{array}$ & $\begin{array}{c}276.4 \\
\text { A }\end{array}$ & $\begin{array}{c}270.0 \\
\text { B }\end{array}$ & & $\begin{array}{c}253.9 \\
\text { C }\end{array}$ & $\begin{array}{c}236.5 \\
\text { D }\end{array}$ & $\begin{array}{c}274.0 \\
\text { B }\end{array}$ & $\begin{array}{c}281.7 \\
\text { A }\end{array}$ & $\begin{array}{c}276.0 \\
\text { AB }\end{array}$ & \\
\hline & \multicolumn{12}{|c|}{ Number of rows per ear } \\
\hline Traditional & 13.33 & 14.00 & 14.67 & 14.67 & 14.00 & $\begin{array}{c}14.13 \\
\text { A }\end{array}$ & 13.33 & 13.33 & 14.00 & 16.00 & 13.33 & $\begin{array}{c}14.00 \\
\text { Ab }\end{array}$ \\
\hline GP1 & 13.33 & 14.67 & 15.33 & 15.33 & 14.00 & $\begin{array}{c}14.53 \\
\text { A }\end{array}$ & 14.00 & 14.00 & 15.33 & 16.00 & 14.00 & $\begin{array}{c}14.67 \\
\text { A }\end{array}$ \\
\hline GP2 & 12.00 & 12.67 & 12.67 & 14.00 & 13.33 & $\begin{array}{c}12.93 \\
\text { B }\end{array}$ & 12.67 & 13.33 & 12.67 & 13.33 & 13.33 & $\begin{array}{c}13.07 \\
\text { BC }\end{array}$ \\
\hline GP3 & 11.33 & 12.67 & 12.00 & 12.67 & 12.00 & $\begin{array}{c}12.13 \\
\text { B }\end{array}$ & 12.00 & 12.00 & 12.67 & 12.67 & 12.00 & $\begin{array}{c}12.27 \\
\text { C }\end{array}$ \\
\hline \multirow[t]{2}{*}{ Mean } & $\begin{array}{c}12.50 \\
\mathrm{C}\end{array}$ & $\begin{array}{c}13.50 \\
\text { AB }\end{array}$ & $\begin{array}{c}13.67 \\
\text { AB }\end{array}$ & $\begin{array}{c}14.17 \\
\text { A }\end{array}$ & $\begin{array}{c}13.33 \\
\text { B }\end{array}$ & & $\begin{array}{c}13.00 \\
\text { B }\end{array}$ & $\begin{array}{c}13.17 \\
\text { B }\end{array}$ & $\begin{array}{c}13.67 \\
\text { B }\end{array}$ & $\begin{array}{c}14.50 \\
\mathrm{~A}\end{array}$ & $\begin{array}{c}13.17 \\
\text { B }\end{array}$ & \\
\hline & \multicolumn{12}{|c|}{ Number of grains per row } \\
\hline Traditional & $\begin{array}{c}42.67 \\
\text { def }\end{array}$ & $\begin{array}{c}43.67 \\
\text { b-e }\end{array}$ & $\begin{array}{c}44.00 \\
\text { b-e }\end{array}$ & $\begin{array}{c}46.33 \\
\text { abc }\end{array}$ & $\begin{array}{l}43.33 \\
\text { cde }\end{array}$ & $\begin{array}{c}44.00 \\
\text { B }\end{array}$ & $\begin{array}{l}43.33 \\
\text { cde }\end{array}$ & $\begin{array}{c}45.33 \\
\text { bcd }\end{array}$ & $\begin{array}{c}43.67 \\
\text { cde }\end{array}$ & $\begin{array}{c}47.33 \\
\text { ab }\end{array}$ & $\begin{array}{c}43.00 \\
\text { de }\end{array}$ & $\begin{array}{c}44.53 \\
\text { B }\end{array}$ \\
\hline GP1 & $\begin{array}{c}42.67 \\
\text { def }\end{array}$ & $\begin{array}{c}47.00 \\
\mathrm{ab}\end{array}$ & $\begin{array}{c}46.00 \\
a-d\end{array}$ & $\begin{array}{c}48.00 \\
\mathrm{a}\end{array}$ & $\begin{array}{c}45.00 \\
\text { a-d }\end{array}$ & $\begin{array}{c}45.73 \\
\text { A }\end{array}$ & $\begin{array}{l}43.67 \\
\text { cde }\end{array}$ & $\begin{array}{c}48.67 \\
\mathrm{a}\end{array}$ & $\begin{array}{c}46.33 \\
\text { abc }\end{array}$ & $\begin{array}{c}49.00 \\
\mathrm{a}\end{array}$ & $\begin{array}{c}44.67 \\
\text { bcd }\end{array}$ & $\begin{array}{c}46.47 \\
\text { A }\end{array}$ \\
\hline GP2 & $\begin{array}{c}36.67 \\
\text { hi }\end{array}$ & $\begin{array}{c}39.67 \\
\text { fgh }\end{array}$ & $\begin{array}{c}32.67 \\
j \mathrm{k}\end{array}$ & $\begin{array}{c}39.00 \\
\text { gh }\end{array}$ & $\begin{array}{c}40.67 \\
\text { efg }\end{array}$ & $\begin{array}{c}37.73 \\
\text { C }\end{array}$ & $\begin{array}{c}38.67 \\
\text { fg }\end{array}$ & $\begin{array}{c}37.00 \\
\text { ghi }\end{array}$ & $\begin{array}{c}35.33 \\
\text { ij }\end{array}$ & $\begin{array}{c}38.33 \\
\text { fgh }\end{array}$ & $\begin{array}{c}41.00 \\
\text { ef }\end{array}$ & $\begin{array}{c}38.07 \\
\text { C }\end{array}$ \\
\hline GP3 & $\begin{array}{c}30.67 \\
\mathrm{k}\end{array}$ & $\begin{array}{c}34.00 \\
\text { ij }\end{array}$ & $\begin{array}{c}29.67 \\
\mathrm{k}\end{array}$ & $\begin{array}{c}31.67 \\
\mathrm{jk}\end{array}$ & $\begin{array}{c}34.67 \\
\text { ij }\end{array}$ & $\begin{array}{c}32.13 \\
\text { D }\end{array}$ & $\begin{array}{c}33.33 \\
\mathrm{jkl}\end{array}$ & $\begin{array}{c}30.67 \\
1\end{array}$ & $\begin{array}{c}32.00 \\
\mathrm{kl}\end{array}$ & $\begin{array}{c}35.67 \\
\text { hij }\end{array}$ & $\begin{array}{c}34.00 \\
j k\end{array}$ & $\begin{array}{c}33.13 \\
\text { D }\end{array}$ \\
\hline \multirow[t]{2}{*}{ Mean } & $\begin{array}{c}38.17 \\
\text { B }\end{array}$ & $\begin{array}{c}41.08 \\
\text { A }\end{array}$ & $\begin{array}{c}38.08 \\
\text { B }\end{array}$ & $\begin{array}{c}41.25 \\
\text { A }\end{array}$ & $\begin{array}{c}40.92 \\
\text { A }\end{array}$ & & $\begin{array}{c}39.75 \\
\text { B }\end{array}$ & $\begin{array}{c}40.42 \\
\text { B }\end{array}$ & $\begin{array}{c}39.33 \\
\text { B }\end{array}$ & $\begin{array}{c}42.58 \\
\mathrm{~A}\end{array}$ & $\begin{array}{c}40.67 \\
\text { B }\end{array}$ & \\
\hline & \multicolumn{12}{|c|}{ 100-grain weight (g.) } \\
\hline Traditional & 32.71 & 30.47 & 31.68 & 33.82 & 34.21 & $\begin{array}{c}32.58 \\
\text { A }\end{array}$ & 33.47 & 31.01 & 32.22 & 34.37 & 34.40 & $\begin{array}{c}33.10 \\
\text { A }\end{array}$ \\
\hline GP1 & 33.06 & 30.77 & 31.81 & 34.40 & 34.66 & $\begin{array}{c}32.94 \\
\text { A }\end{array}$ & 33.66 & 31.57 & 31.97 & 34.56 & 35.06 & $\begin{array}{c}33.37 \\
\text { A }\end{array}$ \\
\hline GP2 & 31.53 & 29.56 & 30.67 & 32.78 & 32.23 & $\begin{array}{c}31.35 \\
\text { B }\end{array}$ & 32.06 & 29.15 & 30.64 & 32.89 & 32.80 & $\begin{array}{c}31.51 \\
\text { B }\end{array}$ \\
\hline GP3 & 28.94 & 28.25 & 29.60 & 30.32 & 30.54 & $\begin{array}{c}29.53 \\
\text { C }\end{array}$ & 29.08 & 28.54 & 28.33 & 31.65 & 31.49 & $\begin{array}{c}29.82 \\
\text { C }\end{array}$ \\
\hline Mean & $\begin{array}{c}31.56 \\
\text { B }\end{array}$ & $\begin{array}{c}29.76 \\
\text { D }\end{array}$ & $\begin{array}{c}30.94 \\
\text { C }\end{array}$ & $\begin{array}{c}32.83 \\
\text { A }\end{array}$ & $\begin{array}{c}32.91 \\
\text { A }\end{array}$ & & $\begin{array}{c}32.07 \\
\text { B }\end{array}$ & $\begin{array}{c}30.07 \\
\text { D }\end{array}$ & $\begin{array}{c}30.79 \\
\text { C }\end{array}$ & $\begin{array}{c}33.37 \\
\text { A }\end{array}$ & $\begin{array}{c}33.44 \\
\text { A }\end{array}$ & \\
\hline
\end{tabular}

determine tolerance to water stress. The interaction significance may be due to the different responses of each maize genotype to the different irrigation regimes as reported by [27] [29] [39]. 
Table 7. Maize productivity and WUE as affected by surface irrigation systems and maize hybrids during 2013 and 2014 seasons.

\begin{tabular}{|c|c|c|c|c|c|c|c|c|c|c|c|c|}
\hline \multirow{2}{*}{$\begin{array}{c}\text { Hybrids } \\
\text { irrigation } \\
\text { systems }\end{array}$} & \multicolumn{6}{|c|}{2013} & \multicolumn{6}{|c|}{2014} \\
\hline & $\begin{array}{c}\text { SC } \\
10\end{array}$ & $\begin{array}{l}\text { SC } \\
130\end{array}$ & $\begin{array}{l}\text { SC } \\
131\end{array}$ & $\begin{array}{c}\text { SC } \\
2031\end{array}$ & $\begin{array}{c}\text { TWC } \\
321\end{array}$ & Mean & $\begin{array}{l}\text { SC } \\
10\end{array}$ & $\begin{array}{l}\text { SC } \\
130\end{array}$ & $\begin{array}{l}\text { SC } \\
131\end{array}$ & $\begin{array}{c}\text { SC } \\
2031\end{array}$ & $\begin{array}{c}\text { TWC } \\
321\end{array}$ & Mean \\
\hline & \multicolumn{12}{|c|}{ Plant grain yield (g.) } \\
\hline Traditional & $\begin{array}{c}158.03 \\
\text { gh }\end{array}$ & $\begin{array}{c}164.56 \\
\mathrm{fg}\end{array}$ & $\begin{array}{c}171.85 \\
\text { def }\end{array}$ & $\begin{array}{c}209.09 \\
\text { a }\end{array}$ & $\begin{array}{c}169.97 \\
\text { ef }\end{array}$ & $\begin{array}{c}174.70 \\
\text { B }\end{array}$ & $\begin{array}{c}170.64 \\
\mathrm{f}\end{array}$ & $\begin{array}{c}172.64 \\
\mathrm{f}\end{array}$ & $\begin{array}{c}181.18 \\
\text { def }\end{array}$ & $\begin{array}{c}206.90 \\
b\end{array}$ & $\begin{array}{c}179.17 \\
\text { def }\end{array}$ & $\begin{array}{c}182.11 \\
\text { B }\end{array}$ \\
\hline GP1 & $\begin{array}{c}172.70 \\
\text { def }\end{array}$ & $\begin{array}{c}175.45 \\
\text { de }\end{array}$ & $\begin{array}{c}188.51 \\
\text { C }\end{array}$ & $\begin{array}{c}213.01 \\
\mathrm{a}\end{array}$ & $\begin{array}{c}179.74 \\
\mathrm{~d}\end{array}$ & $\begin{array}{c}185.88 \\
\text { A }\end{array}$ & $\begin{array}{c}177.48 \\
\text { ef }\end{array}$ & $\begin{array}{c}179.30 \\
\text { def }\end{array}$ & $\begin{array}{c}193.86 \\
\text { c }\end{array}$ & $\begin{array}{c}218.54 \\
\text { a }\end{array}$ & $\begin{array}{c}186.23 \\
\text { cde }\end{array}$ & $\begin{array}{c}191.08 \\
\text { A }\end{array}$ \\
\hline GP2 & $\begin{array}{c}129.58 \\
\mathrm{j}\end{array}$ & $\begin{array}{c}140.09 \\
\text { i }\end{array}$ & $\begin{array}{c}151.69 \\
\mathrm{~h}\end{array}$ & $\begin{array}{c}200.38 \\
b\end{array}$ & $\begin{array}{c}152.40 \\
\mathrm{~h}\end{array}$ & $\begin{array}{c}154.83 \\
\text { C }\end{array}$ & $\begin{array}{c}135.60 \\
\text { i }\end{array}$ & $\begin{array}{c}148.96 \\
\text { gh }\end{array}$ & $\begin{array}{c}159.18 \\
\mathrm{f}\end{array}$ & $\begin{array}{c}190.04 \\
\text { cd }\end{array}$ & $\begin{array}{c}153.07 \\
\text { gh }\end{array}$ & $\begin{array}{c}157.37 \\
\text { C }\end{array}$ \\
\hline GP3 & $\begin{array}{c}110.38 \\
1\end{array}$ & $\begin{array}{c}118.87 \\
\mathrm{k}\end{array}$ & $\begin{array}{c}133.83 \\
\text { ij }\end{array}$ & $\begin{array}{c}165.50 \\
\mathrm{fg}\end{array}$ & $\begin{array}{c}128.86 \\
\mathrm{j}\end{array}$ & $\begin{array}{c}131.49 \\
\text { D }\end{array}$ & $\begin{array}{c}105.93 \\
\mathrm{k}\end{array}$ & $\begin{array}{c}122.34 \\
\mathrm{j}\end{array}$ & $\begin{array}{c}146.59 \\
\mathrm{~h}\end{array}$ & $\begin{array}{c}158.85 \\
g\end{array}$ & $\begin{array}{c}123.57 \\
\mathrm{j}\end{array}$ & $\begin{array}{c}131.46 \\
\text { D }\end{array}$ \\
\hline Mean & $\begin{array}{c}142.67 \\
\text { D }\end{array}$ & $\begin{array}{c}149.74 \\
C\end{array}$ & $\begin{array}{c}161.47 \\
\text { B }\end{array}$ & $\begin{array}{c}197.00 \\
\mathrm{~A}\end{array}$ & $\begin{array}{c}157.74 \\
\text { B }\end{array}$ & & $\begin{array}{c}147.41 \\
D\end{array}$ & $\begin{array}{c}155.81 \\
C\end{array}$ & $\begin{array}{c}170.20 \\
\text { B }\end{array}$ & $\begin{array}{c}193.58 \\
\text { A }\end{array}$ & $\begin{array}{c}160.51 \\
\mathrm{C}\end{array}$ & \\
\hline & \multicolumn{12}{|c|}{ Grain yield $\left(\mathrm{kg} \cdot \mathrm{ha}^{-1}\right)$} \\
\hline Traditional & $\begin{array}{c}8188.6 \\
\text { gh }\end{array}$ & $\begin{array}{c}8816.4 \\
\text { f }\end{array}$ & $\begin{array}{c}9149.3 \\
\text { e }\end{array}$ & $\begin{array}{c}10523.0 \\
\text { b }\end{array}$ & $\begin{array}{c}9254.9 \\
\text { de }\end{array}$ & $\begin{array}{c}9186.4 \\
\text { B }\end{array}$ & $\begin{array}{l}8524.6 \\
\text { fg }\end{array}$ & $\begin{array}{c}9236.4 \\
\text { de }\end{array}$ & $\begin{array}{c}9744.7 \\
\text { c }\end{array}$ & $\begin{array}{c}10394.9 \\
\text { b }\end{array}$ & $\begin{array}{c}8647.4 \\
\mathrm{f}\end{array}$ & $\begin{array}{c}9309.6 \\
\text { B }\end{array}$ \\
\hline GP1 & $\begin{array}{c}8806.6 \\
\mathrm{f}\end{array}$ & $\begin{array}{c}9305.8 \\
\text { de }\end{array}$ & $\begin{array}{c}9808.8 \\
\text { c }\end{array}$ & $\begin{array}{c}11062.6 \\
\text { a }\end{array}$ & $\begin{array}{c}9493.4 \\
\mathrm{~d}\end{array}$ & $\begin{array}{c}9695.4 \\
\text { A }\end{array}$ & $\begin{array}{c}8992.3 \\
\text { e }\end{array}$ & $\begin{array}{c}9451.9 \\
\text { cd }\end{array}$ & $\begin{array}{c}10167.6 \\
\text { b }\end{array}$ & $\begin{array}{c}10911.8 \\
\mathrm{a}\end{array}$ & $\begin{array}{c}9773.5 \\
\text { c }\end{array}$ & $\begin{array}{c}9859.4 \\
\text { A }\end{array}$ \\
\hline GP2 & $\begin{array}{c}6698.6 \\
\mathrm{j}\end{array}$ & $\begin{array}{c}7255.4 \\
\text { i }\end{array}$ & $\begin{array}{c}8439.4 \\
\mathrm{~g}\end{array}$ & $\begin{array}{c}9851.5 \\
\text { c }\end{array}$ & $\begin{array}{c}7947.4 \\
\text { h }\end{array}$ & $\begin{array}{c}8038.5 \\
\text { C }\end{array}$ & $\begin{array}{c}7358.2 \\
j\end{array}$ & $\begin{array}{c}7969.2 \\
\text { hi }\end{array}$ & $\begin{array}{c}8535.6 \\
\text { fg }\end{array}$ & $\begin{array}{c}9728.4 \\
\text { c }\end{array}$ & $\begin{array}{c}8186.9 \\
\text { gh }\end{array}$ & $\begin{array}{c}8355.6 \\
\text { C }\end{array}$ \\
\hline GP3 & $\begin{array}{c}5811.1 \\
1\end{array}$ & $\begin{array}{c}6262.6 \\
\mathrm{k}\end{array}$ & $\begin{array}{c}7258.6 \\
\text { i }\end{array}$ & $\begin{array}{c}8785.2 \\
f\end{array}$ & $\begin{array}{c}6810.7 \\
\mathrm{j}\end{array}$ & $\begin{array}{c}6985.6 \\
\text { D }\end{array}$ & $\begin{array}{c}5834.4 \\
1\end{array}$ & $\begin{array}{c}7035.8 \\
j k\end{array}$ & $\begin{array}{c}7709.3 \\
\text { i }\end{array}$ & $\begin{array}{c}8260.1 \\
\text { gh }\end{array}$ & $\begin{array}{c}6714.2 \\
\mathrm{k}\end{array}$ & $\begin{array}{c}7110.8 \\
\text { D }\end{array}$ \\
\hline Mean & $\begin{array}{c}7376.2 \\
\mathrm{E}\end{array}$ & $\begin{array}{c}7910.0 \\
\text { D }\end{array}$ & $\begin{array}{c}8664.0 \\
\text { B }\end{array}$ & $\begin{array}{c}10055.6 \\
\text { A }\end{array}$ & $\begin{array}{c}8376.6 \\
\text { C }\end{array}$ & & $\begin{array}{c}7677.4 \\
\text { D }\end{array}$ & $\begin{array}{c}8423.3 \\
\text { C }\end{array}$ & $\begin{array}{c}9039.3 \\
\text { B }\end{array}$ & $\begin{array}{c}9823.8 \\
\text { A }\end{array}$ & $\begin{array}{c}8330.5 \\
\text { C }\end{array}$ & \\
\hline & \multicolumn{12}{|c|}{ Stover yield $\left(\mathrm{kg} \cdot \mathrm{ha}^{-1}\right)$} \\
\hline Traditional & $\begin{array}{c}9653.8 \\
\text { ghi }\end{array}$ & $\begin{array}{c}11515.9 \\
\mathrm{e}\end{array}$ & $\begin{array}{c}12827.0 \\
\text { bc }\end{array}$ & $\begin{array}{c}13445.3 \\
\text { a }\end{array}$ & $\begin{array}{c}11870.4 \\
\text { e }\end{array}$ & $\begin{array}{c}11862.5 \\
\text { B }\end{array}$ & $\begin{array}{c}10380.2 \\
\mathrm{f}\end{array}$ & $\begin{array}{c}11538.0 \\
\text { de }\end{array}$ & $\begin{array}{c}11601.1 \\
\mathrm{e}\end{array}$ & $\begin{array}{c}13247.5 \\
\text { b }\end{array}$ & $\begin{array}{c}11468.2 \\
\text { e }\end{array}$ & $\begin{array}{c}11647.0 \\
\text { B }\end{array}$ \\
\hline GP1 & $\begin{array}{c}10116.5 \\
\text { fg }\end{array}$ & $\begin{array}{c}11980.6 \\
\text { de }\end{array}$ & $\begin{array}{c}13330.6 \\
a b\end{array}$ & $\begin{array}{c}13639.0 \\
\mathrm{a}\end{array}$ & $\begin{array}{c}12013.2 \\
\text { de }\end{array}$ & $\begin{array}{c}12216.0 \\
\text { A }\end{array}$ & $\begin{array}{c}10434.2 \\
\mathrm{f}\end{array}$ & $\begin{array}{c}11701.4 \\
\text { de }\end{array}$ & $\begin{array}{c}12501.1 \\
\text { C }\end{array}$ & $\begin{array}{c}13902.2 \\
\text { a }\end{array}$ & $\begin{array}{c}11928.0 \\
\mathrm{~d}\end{array}$ & $\begin{array}{c}12093.4 \\
\text { A }\end{array}$ \\
\hline GP2 & $\begin{array}{c}7585.2 \\
\mathrm{j}\end{array}$ & $\begin{array}{c}9090.0 \\
\text { i }\end{array}$ & $\begin{array}{c}10472.6 \\
\mathrm{f}\end{array}$ & $\begin{array}{c}12535.7 \\
\text { cd }\end{array}$ & $\begin{array}{c}9690.5 \\
\text { gh }\end{array}$ & $\begin{array}{c}9874.8 \\
\text { C }\end{array}$ & $\begin{array}{c}8103.1 \\
\text { i }\end{array}$ & $\begin{array}{c}9692.9 \\
\mathrm{~g}\end{array}$ & $\begin{array}{c}9665.8 \\
\mathrm{~g}\end{array}$ & $\begin{array}{c}11771.8 \\
\text { de }\end{array}$ & $\begin{array}{c}9463.4 \\
\mathrm{~g}\end{array}$ & $\begin{array}{c}9739.4 \\
\text { C }\end{array}$ \\
\hline GP3 & $\begin{array}{c}6841.2 \\
\mathrm{k}\end{array}$ & $\begin{array}{c}7338.7 \\
j k\end{array}$ & $\begin{array}{c}9311.0 \\
\text { hi }\end{array}$ & $\begin{array}{c}9955.7 \\
\text { fg }\end{array}$ & $\begin{array}{c}7651.0 \\
\mathrm{~J}\end{array}$ & $\begin{array}{c}8219.5 \\
\text { D }\end{array}$ & $\begin{array}{c}7193.8 \\
\mathrm{j}\end{array}$ & $\begin{array}{c}7405.7 \\
\mathrm{j}\end{array}$ & $\begin{array}{c}8543.8 \\
\text { h }\end{array}$ & $\begin{array}{c}9541.7 \\
\mathrm{~g}\end{array}$ & $\begin{array}{c}7130.6 \\
\mathrm{j}\end{array}$ & $\begin{array}{c}7963.1 \\
\text { D }\end{array}$ \\
\hline Mean & $\begin{array}{c}8549.2 \\
\text { E }\end{array}$ & $\begin{array}{c}9981.3 \\
\text { D }\end{array}$ & $\begin{array}{c}11485.3 \\
\text { B }\end{array}$ & $\begin{array}{c}12393.9 \\
\text { A }\end{array}$ & $\begin{array}{c}10306.3 \\
\text { C }\end{array}$ & & $\begin{array}{c}9027.8 \\
\text { D }\end{array}$ & $\begin{array}{c}10084.5 \\
C\end{array}$ & $\begin{array}{c}10577.9 \\
\text { B }\end{array}$ & $\begin{array}{c}12115.8 \\
\text { A }\end{array}$ & $\begin{array}{c}9997.6 \\
C\end{array}$ & \\
\hline & \multicolumn{12}{|c|}{ Water use efficiency (kg grains $\mathrm{m}^{-3}$ ) } \\
\hline Traditional & $\begin{array}{c}1.27 \\
1\end{array}$ & $\begin{array}{c}1.37 \\
\mathrm{jk}\end{array}$ & $\begin{array}{c}1.42 \\
\mathrm{ij}\end{array}$ & $\begin{array}{c}1.64 \\
\text { de }\end{array}$ & $\begin{array}{c}1.44 \\
\text { hi }\end{array}$ & $\begin{array}{c}1.43 \\
D\end{array}$ & $\begin{array}{c}1.33 \\
\mathrm{i}\end{array}$ & $\begin{array}{c}1.44 \\
\mathrm{~h}\end{array}$ & $\begin{array}{c}1.52 \\
\mathrm{~g}\end{array}$ & $\begin{array}{c}1.62 \\
\text { ef }\end{array}$ & $\begin{array}{c}1.35 \\
\mathrm{i}\end{array}$ & $\begin{array}{c}1.45 \\
\mathrm{D}\end{array}$ \\
\hline GP1 & $\begin{array}{c}1.60 \\
\text { ef }\end{array}$ & $\begin{array}{c}1.69 \\
\mathrm{~cd}\end{array}$ & $\begin{array}{c}1.78 \\
b\end{array}$ & $\begin{array}{c}2.01 \\
\mathrm{a}\end{array}$ & $\begin{array}{c}1.72 \\
\text { bc }\end{array}$ & $\begin{array}{c}1.76 \\
\mathrm{~A}\end{array}$ & $\begin{array}{c}1.63 \\
\text { ef }\end{array}$ & $\begin{array}{c}1.72 \\
\text { cd }\end{array}$ & $\begin{array}{c}1.84 \\
b\end{array}$ & $\begin{array}{c}1.98 \\
\mathrm{a}\end{array}$ & $\begin{array}{c}1.77 \\
\mathrm{C}\end{array}$ & $\begin{array}{c}1.79 \\
\mathrm{~A}\end{array}$ \\
\hline GP2 & $\begin{array}{c}1.38 \\
\mathrm{ijk}\end{array}$ & $\begin{array}{c}1.49 \\
\text { gh }\end{array}$ & $\begin{array}{c}1.74 \\
\text { bc }\end{array}$ & $\begin{array}{c}2.02 \\
a\end{array}$ & $\begin{array}{c}1.64 \\
\text { de }\end{array}$ & $\begin{array}{c}1.65 \\
\text { B }\end{array}$ & $\begin{array}{c}1.51 \\
\mathrm{~g}\end{array}$ & $\begin{array}{c}1.64 \\
\text { ef }\end{array}$ & $\begin{array}{c}1.75 \\
\mathrm{C}\end{array}$ & $\begin{array}{c}2.00 \\
a\end{array}$ & $\begin{array}{c}1.68 \\
\text { de }\end{array}$ & $\begin{array}{c}1.72 \\
\text { B }\end{array}$ \\
\hline GP3 & $\begin{array}{c}1.32 \\
\mathrm{kl}\end{array}$ & $\begin{array}{c}1.42 \\
\mathrm{ij}\end{array}$ & $\begin{array}{c}1.64 \\
\text { de }\end{array}$ & $\begin{array}{c}1.99 \\
\mathrm{a}\end{array}$ & $\begin{array}{c}1.54 \\
\mathrm{fg}\end{array}$ & $\begin{array}{c}1.58 \\
C\end{array}$ & $\begin{array}{c}1.32 \\
\mathrm{i}\end{array}$ & $\begin{array}{c}1.59 \\
\mathrm{f}\end{array}$ & $\begin{array}{c}1.75 \\
\mathrm{C}\end{array}$ & $\begin{array}{c}1.87 \\
b\end{array}$ & $\begin{array}{c}1.52 \\
\mathrm{~g}\end{array}$ & $\begin{array}{c}1.61 \\
\mathrm{C}\end{array}$ \\
\hline Mean & $\begin{array}{c}1.39 \\
\mathrm{E}\end{array}$ & $\begin{array}{c}1.49 \\
\text { D }\end{array}$ & $\begin{array}{c}1.65 \\
\text { B }\end{array}$ & $\begin{array}{c}1.92 \\
\mathrm{~A}\end{array}$ & $\begin{array}{c}1.59 \\
\mathrm{C}\end{array}$ & & $\begin{array}{c}1.45 \\
\text { D }\end{array}$ & $\begin{array}{c}1.60 \\
\mathrm{C}\end{array}$ & $\begin{array}{c}1.71 \\
\text { B }\end{array}$ & $\begin{array}{c}1.87 \\
\text { A }\end{array}$ & $\begin{array}{c}1.58 \\
\mathrm{C}\end{array}$ & \\
\hline
\end{tabular}

\subsection{Irrigation Water Use Efficiency}

The combined effects of irrigation systems and varieties on irrigation water use efficiency were significant in both seasons (Table 7). GP1 had WUE values that were 5.34\%, 11.29\% and 23.26\% greater than those of GP2, GP3 and traditional methods, respectively. The use of GP1 technique increased WUE from $1.44 \mathrm{~kg} \cdot \mathrm{m}^{-3}$ for the farmer's usual water management practice to $1.78 \mathrm{~kg} \cdot \mathrm{m}^{-3}$ as average of both seasons. Increasing irrigation water led to decrease water use efficiency. Low WUE can occur when soil evaporation is high relative to crop evapo- 
transpiration, early growth rates are low, water application does not correspond to crop demand, or shallow roots are unable to utilize deep water in the soil profile [12]. In this concern, [40] found that using gated pipes to irrigate long furrow (100 m long) resulted in saving water by $20 \%$ and $38 \%$ as well as increasing the water use efficiency by $58 \%$ and $26 \%$ for beans and corn, respectively compared with conventional surface irrigation method used short furrows (6 - $10 \mathrm{~m}$ long) in sandy soil. Meanwhile, [26] stated that use gated pipe system in sugarcane fields can reduce the irrigation quota by $48-156 \mathrm{~m}^{3} \cdot \mathrm{ha}^{-1}$ for each irrigation cycle in comparing with open field head ditch.

The differences among the five maize varieties in their WUE were significant in both seasons. It is clear that S.C 2031 variety recorded the highest values followed by S.C 131. On the contrary, the lowest one was obtained by S.C 10. This means that S.C 2031 variety is more able to extract water from soil zones, and convert it into plant biomass than other varieties. [41] reported that maize plants are especially sensitive to water stress because their root system is relatively sparse.WUE of maize cultivars has been studied by several workers including [42] and [43]. These studies are important for identifying maize cultivars that are efficient in the use of limited soil water for biomass and grain yield production.

The response of maize plants to irrigation systems has been shown to change with hybrids (Table 7). It is interesting to note that the irrigated S.C 2031 variety with GP1 and GP2 system had highest WUE values similar to those obtained by the same variety under GP3 in the first season. The lower WUE was recorded with traditional irrigation especially under planting S.C 10 variety. Better management of the water balance with gated pipes technique can make significant improvements in water use efficiency under maize genotypes. [31] stated that maize genotypes with early adventitious rooting, increase root $\mathrm{NAD}^{+}$-alcohol dehydrogenase activity and high starch accumulation in stem tissues showed good tolerance to excess soil moisture stress.

\subsection{Nitrogen Accumulation}

Plant tissue analysis has been used to reveal the status of nitrogen element in a soil-plant system. The grains and stover nitrogen percentage and accumulations were significantly different with various irrigation regimes and varieties (Table 8). Nitrogen \% and accumulation were significantly increased by $1.30 \%$ and $7.05 \%$ in grains and $2.92 \%$ and $6.33 \%$ in stover, respectively when GP1 was applied in comparing to traditional irrigation. Nitrogen uptake was more dependent on water supply. $\mathrm{N}$ uptake decrease with greater water applied [30]. Since the oxygen is limited, microorganisms may turn to pathways of metabolism that can affect the availability and uptake of certain plant nutrients [29]. Adequate moisture improve uptake of nutrients by diffusion and root interaction, and will increase organic matter decomposition, which releases $\mathrm{N}, \mathrm{P}$ and $\mathrm{S}$. Low moisture can result in the formation of insoluble nutrient-containing. However, flooded or very wet soils increase the solubility of minerals and promote nitrogen leaching and the contamination of ground water by nitrates. [13] stated that maize leaf nutrients concentrations were reduced with increasing applied water quantity, indicating that leaf $\mathrm{N}$ concentration with the $0.80 \mathrm{ET}_{\mathrm{c}}$ treatment were generally equal to or higher than the concentrations with 1.00 $\mathrm{ET}_{\mathrm{c}}$.

By comparing the average values of varieties, the highest nitrogen percent and accumulations were attained by S.C 2031 more than other varieties. Meanwhile, S.C 130, T.W.C 321 and S.C 10 had the lowest values of grain $\mathrm{N}$, stover $\mathrm{N}$ and accumulations, respectively. The canopy nutritional state can be evaluated through pigment concentration, as chlorophyll concentration in leaves is usually correlated to its nitrogen content. Indices that are good indicators of Chl are usually also good indicators of N-content [33]. Selection of genotypes with a more efficient mechanism of $\mathrm{N}$ uptake and metabolism is strategy aimed to increasing maize crop. From these results, it can be concluded that S.C 2031 had the ability to transport enough absorbed nitrogen to grains and stover more than that of other varieties.

Maize varieties differently responded to irrigation systems for grain $\mathrm{N}$ and accumulations (Table 8). It attributed to the differences of root distribution of genotypes under irrigation regimes. The hybrids response, especially S.C 2031, was increased with using GP1. Under this combination, $\mathrm{N}$ accumulations represent the capacity of plants to absorb more $\mathrm{N}$ from soil and fertilization applied. These findings are in line with the results of [1].

\section{Conclusion}

Irrigation management decisions should be made based on the amount of water applied and how this relates to the consumptive use demands of the plants and the soil water holding capacity. Adopting proper irrigation man- 
Table 8. Nitrogen percentage and accumulation as affected by surface irrigation systems and maize hybrids during 2013 and 2014 seasons.

\begin{tabular}{|c|c|c|c|c|c|c|c|c|c|c|c|c|}
\hline \multirow{2}{*}{$\begin{array}{c}\text { Hybrids } \\
\text { irrigation } \\
\text { systems }\end{array}$} & \multicolumn{6}{|c|}{2013} & \multicolumn{6}{|c|}{2014} \\
\hline & $\begin{array}{l}\text { SC } \\
10\end{array}$ & $\begin{array}{l}\text { SC } \\
130\end{array}$ & $\begin{array}{l}\text { SC } \\
131\end{array}$ & $\begin{array}{c}\text { SC } \\
2031\end{array}$ & $\begin{array}{c}\text { TWC } \\
321\end{array}$ & Mean & $\begin{array}{l}\text { SC } \\
10\end{array}$ & $\begin{array}{l}\text { SC } \\
130\end{array}$ & $\begin{array}{l}\text { SC } \\
131\end{array}$ & $\begin{array}{c}\text { SC } \\
2031\end{array}$ & $\begin{array}{c}\text { TWC } \\
321\end{array}$ & Mean \\
\hline & \multicolumn{12}{|c|}{ Grain nitrogen (\%) } \\
\hline Traditional & $\begin{array}{c}1.541 \\
\text { cd }\end{array}$ & $\begin{array}{c}1.519 \\
\text { fgh }\end{array}$ & $\begin{array}{c}1.533 \\
\text { def }\end{array}$ & $\begin{array}{c}1.592 \\
\mathrm{a}\end{array}$ & $\begin{array}{c}1.470 \\
\mathrm{k}\end{array}$ & $\begin{array}{c}1.531 \\
\text { B }\end{array}$ & $\begin{array}{c}1.520 \\
\text { efg }\end{array}$ & $\begin{array}{c}1.536 \\
\mathrm{e}\end{array}$ & $\begin{array}{c}1.581 \\
\mathrm{ab}\end{array}$ & $\begin{array}{c}1.589 \\
\mathrm{a}\end{array}$ & $\begin{array}{c}1.517 \\
\mathrm{fg}\end{array}$ & $\begin{array}{c}1.549 \\
\text { B }\end{array}$ \\
\hline GP1 & $\begin{array}{c}1.563 \\
\mathrm{~b}\end{array}$ & $\begin{array}{c}1.537 \\
\text { de }\end{array}$ & $\begin{array}{c}1.555 \\
\text { bc }\end{array}$ & $\begin{array}{c}1.601 \\
\mathrm{a}\end{array}$ & $\begin{array}{c}1.492 \\
\mathrm{j}\end{array}$ & $\begin{array}{c}1.550 \\
\mathrm{~A}\end{array}$ & $\begin{array}{c}1.571 \\
\text { bc }\end{array}$ & $\begin{array}{c}1.556 \\
\text { cd }\end{array}$ & $\begin{array}{c}1.593 \\
\mathrm{a}\end{array}$ & $\begin{array}{c}1.596 \\
\mathrm{a}\end{array}$ & $\begin{array}{c}1.534 \\
\mathrm{e}\end{array}$ & $\begin{array}{c}1.570 \\
\mathrm{~A}\end{array}$ \\
\hline GP2 & $\begin{array}{c}1.524 \\
\text { efg }\end{array}$ & $\begin{array}{c}1.492 \\
\mathrm{j}\end{array}$ & $\begin{array}{c}1.512 \\
\text { gh }\end{array}$ & $\begin{array}{c}1.547 \\
\text { cd }\end{array}$ & $\begin{array}{c}1.451 \\
\mathrm{l}\end{array}$ & $\begin{array}{c}1.505 \\
\text { C }\end{array}$ & $\begin{array}{c}1.505 \\
\text { gh }\end{array}$ & $\begin{array}{c}1.514 \\
\mathrm{~g}\end{array}$ & $\begin{array}{c}1.569 \\
\text { bcd }\end{array}$ & $\begin{array}{c}1.554 \\
\mathrm{~d}\end{array}$ & $\begin{array}{c}1.472 \\
\mathrm{i}\end{array}$ & $\begin{array}{c}1.523 \\
\text { C }\end{array}$ \\
\hline GP3 & $\begin{array}{c}1.509 \\
\text { hi }\end{array}$ & $\begin{array}{c}1.483 \\
\mathrm{jk}\end{array}$ & $\begin{array}{c}1.496 \\
\text { ij }\end{array}$ & $\begin{array}{c}1.525 \\
\text { efg }\end{array}$ & $\begin{array}{c}1.436 \\
\mathrm{~m}\end{array}$ & $\begin{array}{c}1.490 \\
\mathrm{C}\end{array}$ & $\begin{array}{c}1.492 \\
h\end{array}$ & $\begin{array}{c}1.455 \\
\mathrm{j}\end{array}$ & $\begin{array}{c}1.456 \\
\mathrm{j}\end{array}$ & $\begin{array}{c}1.532 \\
\text { ef }\end{array}$ & $\begin{array}{c}1.441 \\
\mathrm{j}\end{array}$ & $\begin{array}{c}1.475 \\
\text { D }\end{array}$ \\
\hline \multirow[t]{2}{*}{ Mean } & $\begin{array}{c}1.534 \\
\text { B }\end{array}$ & $\begin{array}{c}1.508 \\
\mathrm{D}\end{array}$ & $\begin{array}{c}1.524 \\
\text { C }\end{array}$ & $\begin{array}{c}1.566 \\
\mathrm{~A}\end{array}$ & $\begin{array}{c}1.462 \\
E\end{array}$ & & $\begin{array}{c}1.522 \\
\text { C }\end{array}$ & $\begin{array}{c}1.515 \\
\mathrm{C}\end{array}$ & $\begin{array}{c}1.550 \\
\text { B }\end{array}$ & $\begin{array}{c}1.568 \\
\mathrm{~A}\end{array}$ & $\begin{array}{c}1.491 \\
\mathrm{D}\end{array}$ & \\
\hline & \multicolumn{12}{|c|}{ Nitrogen accumulation in grain $\left(\mathrm{kg} \cdot \mathrm{ha}^{-1}\right)$} \\
\hline Traditional & $\begin{array}{c}126.2 \\
\mathrm{~h}\end{array}$ & $\begin{array}{c}133.9 \\
\mathrm{~g}\end{array}$ & $\begin{array}{c}140.3 \\
\text { def }\end{array}$ & $\begin{array}{c}167.5 \\
\text { b }\end{array}$ & $\begin{array}{c}136.0 \\
\text { fg }\end{array}$ & $\begin{array}{c}140.8 \\
\text { B }\end{array}$ & $\begin{array}{c}129.6 \\
\text { gh }\end{array}$ & $\begin{array}{c}141.9 \\
\text { ef }\end{array}$ & $\begin{array}{c}154.1 \\
\text { c }\end{array}$ & $\begin{array}{c}165.2 \\
\mathrm{~b}\end{array}$ & $\begin{array}{c}131.2 \\
\text { gh }\end{array}$ & $\begin{array}{c}144.4 \\
\text { B }\end{array}$ \\
\hline GP1 & $\begin{array}{c}137.7 \\
\text { efg }\end{array}$ & $\begin{array}{c}143.0 \\
\mathrm{~d}\end{array}$ & $\begin{array}{c}152.5 \\
\text { c }\end{array}$ & $\begin{array}{c}177.1 \\
\mathrm{a}\end{array}$ & $\begin{array}{c}141.6 \\
\text { de }\end{array}$ & $\begin{array}{c}150.4 \\
\text { A }\end{array}$ & $\begin{array}{c}141.2 \\
\mathrm{f}\end{array}$ & $\begin{array}{c}147.1 \\
\text { de }\end{array}$ & $\begin{array}{c}162.0 \\
\text { b }\end{array}$ & $\begin{array}{c}174.1 \\
\mathrm{a}\end{array}$ & $\begin{array}{c}149.9 \\
\text { cd }\end{array}$ & $\begin{array}{c}154.9 \\
\text { A }\end{array}$ \\
\hline GP2 & $\begin{array}{c}102.1 \\
\mathrm{k}\end{array}$ & $\begin{array}{c}108.3 \\
j\end{array}$ & $\begin{array}{c}127.6 \\
\mathrm{~h}\end{array}$ & $\begin{array}{c}152.4 \\
\text { c }\end{array}$ & $\begin{array}{c}115.3 \\
\mathrm{i}\end{array}$ & $\begin{array}{c}121.1 \\
C\end{array}$ & $\begin{array}{c}110.8 \\
\mathrm{j}\end{array}$ & $\begin{array}{c}120.6 \\
\text { i }\end{array}$ & $\begin{array}{c}133.9 \\
\text { g }\end{array}$ & $\begin{array}{c}151.2 \\
\text { cd }\end{array}$ & $\begin{array}{c}120.5 \\
\mathrm{i}\end{array}$ & $\begin{array}{c}127.4 \\
\text { C }\end{array}$ \\
\hline GP3 & $\begin{array}{c}87.7 \\
\mathrm{~m}\end{array}$ & $\begin{array}{c}92.9 \\
1\end{array}$ & $\begin{array}{c}108.6 \\
j\end{array}$ & $\begin{array}{c}134.0 \\
\mathrm{~g}\end{array}$ & $\begin{array}{c}97.8 \\
\mathrm{k}\end{array}$ & $\begin{array}{c}104.2 \\
\text { D }\end{array}$ & $\begin{array}{c}87.0 \\
\mathrm{~m}\end{array}$ & $\begin{array}{c}102.4 \\
\mathrm{k}\end{array}$ & $\begin{array}{c}112.2 \\
\mathrm{j}\end{array}$ & $\begin{array}{c}126.5 \\
\mathrm{~h}\end{array}$ & $\begin{array}{c}96.8 \\
1\end{array}$ & $\begin{array}{c}105.0 \\
\text { D }\end{array}$ \\
\hline Mean & $\begin{array}{c}113.4 \\
\text { E }\end{array}$ & $\begin{array}{c}119.5 \\
\text { D }\end{array}$ & $\begin{array}{c}132.2 \\
\text { B }\end{array}$ & $\begin{array}{c}157.8 \\
\text { A }\end{array}$ & $\begin{array}{c}122.7 \\
\text { C }\end{array}$ & & $\begin{array}{c}117.2 \\
\text { E }\end{array}$ & $\begin{array}{c}128.0 \\
C\end{array}$ & $\begin{array}{c}140.5 \\
\text { B }\end{array}$ & $\begin{array}{c}154.2 \\
\text { A }\end{array}$ & $\begin{array}{c}124.6 \\
\text { D }\end{array}$ & \\
\hline & \multicolumn{12}{|c|}{ Stover nitrogen $(\%)$} \\
\hline Traditional & 0.759 & 0.723 & 0.758 & 0.771 & 0.685 & $\begin{array}{c}0.739 \\
\text { A }\end{array}$ & 0.733 & 0.734 & 0.764 & 0.773 & 0.663 & $\begin{array}{c}0.733 \\
\text { B }\end{array}$ \\
\hline GP1 & 0.767 & 0.763 & 0.769 & 0.775 & 0.699 & $\begin{array}{c}0.755 \\
\text { A }\end{array}$ & 0.773 & 0.751 & 0.780 & 0.798 & 0.699 & $\begin{array}{c}0.760 \\
\text { A }\end{array}$ \\
\hline GP2 & 0.761 & 0.698 & 0.754 & 0.765 & 0.676 & $\begin{array}{c}0.731 \\
\text { A }\end{array}$ & 0.742 & 0.678 & 0.734 & 0.768 & 0.682 & $\begin{array}{c}0.721 \\
\text { B }\end{array}$ \\
\hline GP3 & 0.728 & 0.677 & 0.652 & 0.743 & 0.645 & $\begin{array}{c}0.689 \\
\text { B }\end{array}$ & 0.717 & 0.654 & 0.691 & 0.735 & 0.678 & $\begin{array}{c}0.695 \\
\text { C }\end{array}$ \\
\hline \multirow[t]{2}{*}{ Mean } & $\begin{array}{c}0.754 \\
\text { A }\end{array}$ & $\begin{array}{c}0.715 \\
\text { B }\end{array}$ & $\begin{array}{c}0.733 \\
\text { AB }\end{array}$ & $\begin{array}{c}0.764 \\
\text { A }\end{array}$ & $\begin{array}{c}0.676 \\
\text { C }\end{array}$ & & $\begin{array}{c}0.741 \\
\text { AB }\end{array}$ & $\begin{array}{c}0.704 \\
\text { BC }\end{array}$ & $\begin{array}{c}0.742 \\
\text { AB }\end{array}$ & $\begin{array}{c}0.769 \\
\text { A }\end{array}$ & $\begin{array}{c}0.681 \\
C\end{array}$ & \\
\hline & \multicolumn{12}{|c|}{ Nitrogen accumulation in stover $\left(\mathrm{kg} \cdot \mathrm{ha}^{-1}\right)$} \\
\hline Traditional & $\begin{array}{c}73.3 \\
\mathrm{f}\end{array}$ & $\begin{array}{c}83.3 \\
\mathrm{e}\end{array}$ & $\begin{array}{l}97.2 \\
\text { bcd }\end{array}$ & $\begin{array}{c}103.7 \\
\text { ab }\end{array}$ & $\begin{array}{c}81.3 \\
\mathrm{e}\end{array}$ & $\begin{array}{c}87.8 \\
\text { B }\end{array}$ & $\begin{array}{c}76.1 \\
\text { ef }\end{array}$ & $\begin{array}{c}84.7 \\
\text { cd }\end{array}$ & $\begin{array}{c}88.6 \\
\text { c }\end{array}$ & $\begin{array}{c}102.4 \\
\mathrm{~b}\end{array}$ & $\begin{array}{c}76.0 \\
\text { ef }\end{array}$ & $\begin{array}{c}85.6 \\
\text { B }\end{array}$ \\
\hline GP1 & $\begin{array}{c}77.6 \\
\text { ef }\end{array}$ & $\begin{array}{c}91.4 \\
\mathrm{~d}\end{array}$ & $\begin{array}{c}102.5 \\
\text { abc }\end{array}$ & $\begin{array}{c}105.7 \\
\mathrm{a}\end{array}$ & $\begin{array}{c}84.0 \\
\mathrm{e}\end{array}$ & $\begin{array}{c}92.2 \\
\text { A }\end{array}$ & $\begin{array}{c}80.7 \\
\text { de }\end{array}$ & $\begin{array}{c}87.9 \\
\text { cd }\end{array}$ & $\begin{array}{c}97.5 \\
\mathrm{~b}\end{array}$ & $\begin{array}{c}110.9 \\
\text { a }\end{array}$ & $\begin{array}{l}83.4 \\
\text { cde }\end{array}$ & $\begin{array}{c}92.1 \\
\text { A }\end{array}$ \\
\hline GP2 & $\begin{array}{c}57.7 \\
\mathrm{~h}\end{array}$ & $\begin{array}{c}63.5 \\
\text { gh }\end{array}$ & $\begin{array}{c}79.0 \\
\text { ef }\end{array}$ & $\begin{array}{c}95.9 \\
\text { cd }\end{array}$ & $\begin{array}{c}65.5 \\
\mathrm{~g}\end{array}$ & $\begin{array}{c}72.3 \\
\mathrm{C}\end{array}$ & $\begin{array}{c}60.0 \\
\mathrm{~h}\end{array}$ & $\begin{array}{c}66.0 \\
\text { gh }\end{array}$ & $\begin{array}{c}70.9 \\
\text { fg }\end{array}$ & $\begin{array}{c}90.5 \\
\text { c }\end{array}$ & $\begin{array}{c}64.5 \\
\text { gh }\end{array}$ & $\begin{array}{c}70.4 \\
\text { C }\end{array}$ \\
\hline GP3 & $\begin{array}{c}49.8 \\
\mathrm{i}\end{array}$ & $\begin{array}{c}49.7 \\
\mathrm{i}\end{array}$ & $\begin{array}{c}60.7 \\
\text { gh }\end{array}$ & $\begin{array}{c}74.2 \\
\mathrm{f}\end{array}$ & $\begin{array}{c}49.4 \\
\mathrm{i}\end{array}$ & $\begin{array}{c}56.7 \\
\text { D }\end{array}$ & $\begin{array}{c}51.6 \\
\mathrm{i}\end{array}$ & $\begin{array}{c}48.4 \\
\mathrm{i}\end{array}$ & $\begin{array}{c}59.0 \\
\mathrm{~h}\end{array}$ & $\begin{array}{c}70.1 \\
\text { fg }\end{array}$ & $\begin{array}{c}48.3 \\
\mathrm{i}\end{array}$ & $\begin{array}{c}55.5 \\
\text { D }\end{array}$ \\
\hline Mean & $\begin{array}{c}64.6 \\
\text { D }\end{array}$ & $\begin{array}{c}72.0 \\
\mathrm{C}\end{array}$ & $\begin{array}{c}84.9 \\
\text { B }\end{array}$ & $\begin{array}{c}94.8 \\
\text { A }\end{array}$ & $\begin{array}{c}70.0 \\
\text { C }\end{array}$ & & $\begin{array}{c}67.1 \\
\text { C }\end{array}$ & $\begin{array}{c}71.7 \\
\text { C }\end{array}$ & $\begin{array}{c}79.0 \\
\text { B }\end{array}$ & $\begin{array}{c}93.5 \\
\text { A }\end{array}$ & $\begin{array}{c}68.1 \\
C\end{array}$ & \\
\hline
\end{tabular}

agement strategies can limit negative impacts. Using a higher efficiency gated pipes irrigation system is recommended for irrigating maize, especially under deficit irrigation in case of water scarcity from a water-saving viewpoint. Selection of maize genotypes that have more efficient water use this will affect positively on agricultural production in general and in particular maize crop. From this study, we can conclude that substantial amounts of water can be saved by applying GP1 $\left(5500 \mathrm{~m}^{3} \cdot \mathrm{ha}^{-1}\right)$ with significant increases in yield especially with sowing the new variety (S.C 2031) where it found that their impacts were positive on water use efficiency 
and productivity under old lands conditions.

\section{Acknowledgements}

The authors are grateful to staff of National Laboratory for Testing Irrigation Equipment for their precious support through laboratory work.

\section{References}

[1] Moser, S.B. (2004) Effects of Pre-Anthesis Drought Stress and Nitrogen on Yield, Nitrogen Use Efficiency, and Grain Minerals of Tropical Maize Varieties. Ph.D. Thesis, Swiss Federal Institute of Technology, Zurich.

[2] Adebo, F.A. and Olaoye, G. (2010) Growth Indices and Grain Yield Attributes in Six Maize Cultivars Representing Two Era of Maize Breeding in Nigeria. Journal of Agricultural Sciences, 2, 218-228. http://dx.doi.org/10.5539/jas.v2n3p218

[3] Mohamed, N.A. (2004) Principal Component and Response Curve Analysis of Some Maize Hybrids to Different Nitrogen Fertilization Levels and Plant Density. Bulletin of the Faculty of Science of Cairo University, 55, 531-556.

[4] El-Gizawy, N.K.B. and Salem, H.M. (2010) Influence of Nitrogen Sources on Yield and Its Components of Some Maize Varieties. World Journal of Agricultural Sciences, 6, 218-223.

[5] Abdel-Maksoud, M.F. and Sarhan, A.A. (2008) Response of Some Maize Hybrids to Bio and Chemical Nitrogen Fertilization. Zagazig Journal of Agricultural Research, 35, 497-515.

[6] Chaves, M.M., Pereira, J.S., Maroco, J., Rodrigues, M.L., Ricardo, C.P., Osorio, L.M., Carvalho, I., Faria, T. and Pinheiro, C. (2002) How Plants Cope with Water Stress in the Field. Photosynthesis and Growth. Annals of Botany, 89, 907-916. http://dx.doi.org/10.1093/aob/mcf105

[7] Asharaf, M. (2010) Inducing Drought Tolerance in Plants: Recent Advances. Biotechnology Advances, 28, $169-183$. http://dx.doi.org/10.1016/j.biotechadv.2009.11.005

[8] El-Quosy, D. (2011) Water Resources in Egypt: Availability and Allocation. Proceedings of the Egypt-AustraliaICARDA Workshop on Farm Water Use Efficiency, Cairo, 26-29 July 2011, 9.

[9] Abou Kheira, A.A. (2009) Comparison among Different Irrigation Systems for Deficit-Irrigated Corn in the Nile Valley. Agricultural Engineering International: CIGR Journal, 14, 1-25.

[10] Amer, K.H. (2007) Surface Irrigation Evaluation Based on Analytical Interrelation among Water Infiltration, Advance and Recession. Proceeding of the Irrigation Association, San Diego, 9-11 December 2007, 433-445.

[11] Karrou, M., Oweis, T., El Enein, R.A. and Sherif, M. (2012) Yield and Water Productivity of Maize and Wheat under Deficit and Raised Bed Irrigation Practices in Egypt. African Journal of Agricultural Research, 7, 1755-1760.

[12] El-Hendawy, S.E., Abd El-Lattief, E.A., Ahmed, M.S. and Schmidhalter, U. (2008) Irrigation Rate and Plant Density Effects on Yield and Water Use Efficiency of Drip-Irrigated Corn. Agricultural Water Management, 95, 836-844. http://dx.doi.org/10.1016/j.agwat.2008.02.008

[13] Al-Kaisi, M.M. and Yin, X. (2003) Effects of Nitrogen Rate, Irrigation Rate, and Plant Population on Corn Yield and Water Use Efficiency. Agronomy Journal, 95, 1475-1482. http://dx.doi.org/10.2134/agronj2003.1475

[14] Albertson, M.L., Barton, J.R. and Simons, D.B. (1960) Fluid Mechanics for Engineers. Prentice-Hall, Inc., Englewood Cliffs.

[15] Jackson, M.L. (1973) Soil Chemical Analysis. Prentice Hall of India, Ltd., New Delhi.

[16] Chapman, H.D. and Pratt, P.F. (1978) Methods of Analysis for Soils, Plants and Water. Division of Agriculture Sciences, University of California, Davis.

[17] Grossmann, R.B. and Reinsch, T.G. (2002) Bulk Density and Linear Extensibility. In: Dane, J.H. and Topp, C., Eds., Methods of Soil Analysis. Part 4. Physical Methods, SSSA Book Series, No. 5, Soil Science Society of America, Madison, 201-228.

[18] Cassel, D.K. and Nielsen, D.R. (1986) Field Capacity and Available Water Capacity. In: Klute, A., Ed., Methods of Soil Analysis. Part I. Physical and Mineralogical Methods, Agronomy Monograph No. 9, Soil Science Society of America, Madison, 901-926.

[19] Foroud, N., George, E.S. and Entz, T. (1996) Determination of Infiltration Rate from Border Irrigation Advance and Recession Trajectories. Agricultural Water Management, 30, 133-142. http://dx.doi.org/10.1016/0378-3774(95)01222-2

[20] Amer, A.M. and Amer, K.H. (2010) Surface Irrigation Management in Relation to Water Infiltration and Distribution in Soils. Soil and Water Research, 5, 75-87. 
[21] Amer, K.H. (2010) Corn Crop Response under Managing Different Irrigation and Salinity Levels. Agricultural Water Management, 97, 1553-1563. http://dx.doi.org/10.1016/j.agwat.2010.05.010

[22] AACC (2000) Approved Methods of the AACC. $10^{\text {th }}$ Edition, American Association of Cereal Chemists, St. Paul.

[23] Snedecor, G.W. and Cochran, W.G. (1980) Statistical Methods. $7^{\text {th }}$ Edition, The Iowa State University Press, Ames.

[24] Duncan, D.B. (1955) Multiple Range and Multiple F. Tests. Biometrics, 11, 1-42. http://dx.doi.org/10.2307/3001478

[25] Mohammed, A.S.H. (2008) Engineering Studies on Developing the Gated Pipes for Surface Irrigation in Small Holdings. Ph.D. Thesis, Faculty of Agriculture, Minufiya University, Egypt.

[26] Abdel Wahab, D.M. and Adeeb, A.M. (2014) Comparison between Hydro-Flume and Open Field Head Ditch Irrigation Systems at Kenana Sugar Scheme, Sudan. Agricultural Sciences, 5, 588-603. http://dx.doi.org/10.4236/as.2014.57062

[27] Lone, A.A. and Warsi, M.Z.K. (2009) Response of Maize (Zea mays L.) to Excess Soil Moisture (ESM) Tolerance at Different Stages of Life Cycle. Botany Research International, 2, 211-217.

[28] Igbadun, H.E. and Oyebode, M.A. (2000) Effect of Delayed Irrigation at Critical Growth Stage on Yield of Wheat. Journal of Savannah Agricultural Mechanization, 2, 63-64.

[29] Irmak, S. (2014) Plant Growth and Yield as Affected by Wet Soil Conditions Due to Flooding or Over-Irrigation. University of Nebraska_Lincoln Extension. http://www.ianrpubs.unl.edu

[30] Pandey, P.K., Maranville, J.W. and Chetima, M.M. (2000) Deficit Irrigation and Nitrogen Effects on Maize in a Sahelian Environment: II. Shoot Growth, Nitrogen Uptake and Water Extraction. Agricultural Water Management, 46, 1527. http://dx.doi.org/10.1016/S0378-3774(00)00074-3

[31] Zaidi, P.H., Rafique, S. and Singh, N.N. (2003) Response of Maize (Zea mays L.) Genotypes to Excess Soil Moisture Stress: Morpho-Physiological Effects and Basis of Tolerance. European Journal of Agronomy, 19, 383-399. http://dx.doi.org/10.1016/s1161-0301(02)00090-4

[32] Zaidi, P.H., Srinivasan, G. and Sanchez, C. (2003) Morpho-Physiological Traits Associated with Variable Field Performance of Different Types Maize Germplasm across Multiple Environments. Maydica, 48, 207-220.

[33] Bort, J., Araus, J.L. and Casadesús, J. (2005) Physiologically Related Tools for the Assessment of Irrigation Systems Performance Monitoring Water Status in Crop Plants. In: Lamaddalena, N., Lebdi, F., Todorovic, M. and Bogliotti, C., Eds., Irrigation Systems Performance, CIHEAM, Bari, 251-263.

[34] Andrade, F.H., Cirilo, A.G. and Echarte, L. (2000) Factors Affecting Kernel Number in Maize. In: Otegui, M.E. and Slafer, G.A. Eds., Physiological Bases for Maize Improvement, Food Products Press, Binghamton, 59-74.

[35] Athar, H.R. and Ashraf, M. (2009) Strategies for Crop Improvement against Salinity and Drought Stress: An Overview. Tasks for Vegetation Sciences, 44, 1-16. http://dx.doi.org/10.1007/978-1-4020-9065-3_1

[36] Abo Soliman, M.S.M., Osman, H.E., Said, M.M. and Omar, E.H. (2005) Maize, Barley Production and Water Use Efficiency as Influenced by Different Irrigation Methods in Egyptian Old Land. Proceedings of the $13^{\text {th }}$ Annual Conference of the Misr Society of Agricultural Engineering, Cairo, 14-15 December 2005, 1-22.

[37] Abo-Shetaia, A.M.A., Abdel-Gawad, A.A., Mahgoub, G.M.A. and El-Koomy, M.B.A. (2000) Effect of Inter and Intra Ridge Distance between Plants on Yield and Yield Components of Four Yellow Maize Hybrids (Zea mays L.). Arab Universities Journal of Agricultural Sciences, 8, 647-662.

[38] El-Danasoury, M.A.M. (2009) Studies on Some Factors Affecting the Productivity of Maize in New Soil. Ph.D. Thesis, Faculty of Agriculture, Al-Azhar University, Egypt.

[39] Grzesiak, M.T., Marcińska, I., Janowiak, F., Rzepka, A. and Hura, T. (2012) The Relationship between Seedling Growth and Grain Yield under Drought Conditions in Maize and Triticale Genotypes. Acta Physiologiae Plantarum, 34, 1765. http://dx.doi.org/10.1007/s11738-012-1039-2

[40] Rady, M.A. (1993) Project Evaluation of Irrigation System on Sandy and Calcareous Soils. Academy of Scientific Research and Technology (ASRT), Cairo.

[41] Ogola, J.B.O., Wheeler, T.R. and Harris, P.M. (2002) Effects of Nitrogen and Irrigation on Water Use of Maize. Field Crops Research, 78, 105-117. http://dx.doi.org/10.1016/S0378-4290(02)00116-8

[42] Asare, D.K., Frimpong, J.O., Ayeh, E.O. and Amoatey, H.M. (2011) Water Use Efficiencies of Maize Cultivars Grown under Rain-Fed Conditions. Agricultural Sciences, 2, 125-130. http://dx.doi.org/10.4236/as.2011.22018

[43] Bu, L., Chen, X., Li, S., Liu, J., Zhu, L., Luo, S., Hill, R. and Zhao, Y. (2015) The Effect of Adapting Cultivars on the Water Use Efficiency of Dryland Maize (Zea mays L.) in Northwestern China. Agricultural Water Management, 138, 1-9. http://dx.doi.org/10.1016/j.agwat.2014.09.010 